\title{
Pascal, de la polémique à l'apologie
}

\section{Anne Régent}

\section{OpenEdition}

\section{Journals}

Édition électronique

URL : http://journals.openedition.org/ccibp/526

DOI : $10.4000 /$ ccibp. 526

ISSN : 2493-7460

\section{Éditeur}

Centre international Blaise Pascal

\section{Édition imprimée}

Date de publication : 4 décembre 2003

Pagination : 5-21

ISBN : 2-84516-244-8

ISSN : 0249-6674

\section{Référence électronique}

Anne Régent, « Pascal, de la polémique à l'apologie», Courrier du Centre international Blaise Pascal [En ligne], 25 | 2003, mis en ligne le 02 décembre 2015, consulté le 20 avril 2019. URL : http:// journals.openedition.org/ccibp/526 ; DOI : 10.4000/ccibp.526

Ce document a été généré automatiquement le 20 avril 2019

Centre international Blaise Pascal 


\title{
Pascal, de la polémique à l'apologie
}

\author{
Anne Régent
}

«Tout auteur a un sens auquel tous les passages contraires s'accordent ou il n'a point de sens du

tout 》

Pascal, Pensées, fragment 257-289

Rupture ou continuité ? Le problème de l'articulation entre les deux œuvres pascaliennes majeures, Les Provinciales et les Pensées, correspondant à deux formes d'écriture que l'anachronisme nous interdit de qualifier d'engagées, a suscité les développements les plus contradictoires, peut-être parce qu'il n'a jamais été abordé pour lui-même. En posant quelques jalons permettant de préciser la manière dont Pascal passe d'un projet à l'autre, nous ne prétendrons bien entendu nullement apporter à la question ambitieusement - présomptueusement? - posée par le titre de cet article, une réponse exhaustive et définitive; il s'agira simplement de se demander comment Pascal effectue dès le début de la rédaction des Pensées un retour d'une extraordinaire fécondité sur la querelle des Provinciales alors encore en cours, de s'interroger sur la manière dont, à partir d'une dispute théologique apparemment réservée à des spécialistes, il élabore très tôt une pensée apologétique remarquablement cohérente, se déployant dans de multiples directions anthropologiques.

2 Le lien philologique entre deux textes apparemment aussi différents est assez évident. On sait que de nombreux fragments rassemblés et publiés par les éditeurs sous le nom de Pensées sont directement et explicitement liés, à des titres divers, à la controverse avec les jésuites ${ }^{1}$. Ces fragments polémiques, soit concernent la querelle des Provinciales, soit ont manifestement servi à Pascal de notes préparatoires à certaines « petites lettres » ellesmêmes. Lorsque le lien avec les Provinciales est aussi net, il représente un indice capital pour la datation des fragments considérés: Pascal réagissant aux différents événements de la polémique, les précisions chronologiques que nous possédons sur le déroulement de celle-ci fournissent de précieux renseignements sur la datation des fragments qui s'y rapportent. 
3 Ainsi, le fragment 956-791 compte de nombreux passages repris presque mot pour mot dans les premières lettres, dont il constitue très vraisemblablement un « avant-texte ». De même, la dénonciation ad hominem des Provinciales: "Cette proposition serait catholique dans une autre bouche; ce n'est que dans M. Arnauld que la Sorbonne l'a condamnée ${ }^{2}$ ” apparaissait déjà sous une forme plus générale et plus nettement paradoxale dans le «brouillon» inséré dans les Pensées: «Cette proposition est bonne dans un auteur et méchante dans un autre ${ }^{3} »$. Tels fragments :

Vous êtes mauvais politiques. ${ }^{4}$

Gens sans parole, sans foi, sans honneur, sans vérité, doubles de cœur ${ }^{5} .$.

ne sont rien d'autre que des notes utilisées dans les $\mathrm{XII}^{\mathrm{e}}$ et $\mathrm{XIII}^{\mathrm{e}}$ Provinciales: leur rédaction est donc antérieure à la XII lettre, datée du 9 septembre 1656. Quant aux trois dossiers sur les miracles ${ }^{6}$, on situe leur rédaction entre septembre 1656 et novembre $1657^{7}$ . Il est en outre assez remarquable que la majorité des fragments classés ait été rédigée en 1658, alors que la série des Écrits des Curés de Paris continuait le combat des Provinciales contre la morale et la polémique des jésuites. Les contextes de rédaction des deux œuvres sont donc étroitement liés.

4 Cette imbrication chronologique et génétique n'est pas sans rendre problématique l'interprétation de certains fragments, comme le pressentait déjà Nicole :

J'y [dans « l'écrit de M. Pascal»] ai trouvé un grand nombre de pierres assez bien taillées et capables d'orner un grand bâtiment, mais le reste ne m'a paru que des matériaux confus, sans que je visse assez l'usage qu'il voulait en faire. ${ }^{8}$

De fait, si la plupart des fragments essentiellement polémiques se donnent ouvertement pour tels, il demeure des cas douteux, lesquels témoignent de l'entremêlement complexe des deux projets et rendent problématique la «rupture idéologique » entre les deux œuvres postulée par L.Goldmann, opposant dans Le Dieu caché "l'intellectualisme centriste » ou «mi-rationalisme implicite » des Provinciales à «l'extrémisme tragique » des Pensées 9 . On sait qu'à cet effet, il affirme qu'une crise majeure aurait ébranlé les certitudes de Pascal en 1657, à la suite de la condamnation des cinq propositions par le pape, entraînant l'arrêt des Provinciales et conduisant le catholique qu'est Pascal à un dilemme insoluble. Cette crise aurait dépassé en ampleur et en importance les crises (pourtant plus avérées) de 1646 et 1654. Pascal aurait alors rompu avec le thomisme arnaldien pour adopter le pessimisme beaucoup plus radical de Barcos, qui nie implicitement la possibilité d'un domaine de vérité ou d'action intra-mondaine valable. Cependant, L. Goldmann admet lui-même qu'aucun biographe de Pascal n'a jamais remarqué l'existence d'une « crise de 1657 », qui semble bien supposée pour les besoins de la cause.

6 Pour un critique tel que H. Gouhier au contraire, l'Apologie naît de la rencontre entre deux projets : un combat contre des catholiques relâchés, et un combat contre des libertins. L'inspiration demeurerait donc la même ${ }^{10}$, et changerait seulement d'objet: en témoignent les grandes feuilles de 1657 où des notes sur Montaigne, sur Miton et sur leurs prédécesseurs pyrrhoniens voisinent avec des fragments relatifs à la perpétuité de la foi, aux miracles et aux erreurs des jésuites ${ }^{11}$. C'est bien cette jonction, plus facile à supposer théoriquement qu'à cerner en pratique, qui fait l'objet de la présente étude, jonction par laquelle les deux projets se complètent pour donner naissance à une argumentation apologétique moderne, novatrice à bien des égards.

7 Dans cette perspective, l'étude détaillée des fragments rédigés à l'époque des Provinciales est particulièrement précieuse, dans la mesure où elle permet de mieux cerner la 
naissance et la formation du projet apologétique pascalien au rythme d'une querelle qui pourrait lui sembler étrangère. En effet, on le sait, les Provinciales et les Pensées sont partiellement contemporaines, leurs périodes de rédaction se superposant pendant un an, de l'été 1656 à l'été 1657 environ. Cette année correspond à une période cruciale de la vie de Pascal, au cours de laquelle une démarche essentiellement polémique engendre un projet d'une toute autre nature, passage de la querelle à l'apologie qui correspond également à un passage d'un public exclusivement catholique à un public beaucoup plus hétérogène. A ce titre, les travaux de P. Ernst visant à la reconstitution des feuillets originaux des Pensées (avant le découpage et le classement effectués par Pascal en 1658) à partir notamment de l'étude des filigranes du papier, revêtent une importance toute particulière, dans la mesure où ils permettent de déterminer quels sont les fragments dont la date de rédaction est contemporaine des Provinciales $^{12}$. Parmi les quarante-quatre types de papier identifiés par P. Ernst apparaissent huit rames correspondant à des strates successives de rédaction, qui portent environ $75 \%$ des fragments classés ${ }^{13}$.

8 La rame qui correspond à la période de rédaction la plus ancienne, rame utilisée pour les Provinciales elles-mêmes, correspond à une strate mineure ${ }^{14}$, utilisée à partir de septembre-octobre 1656, soit à l'époque de la XII lettre. P. Ernst la désigne par l'appellation quelque peu ésotérique (correspondant à son filigrane) de « Cadran $1 \mathrm{~B}$ cœur $C$ ». Elle comprend essentiellement des textes relatifs au miracle de la Sainte-Épine, des notes préparatoires à la XV Provinciale datée du 25 novembre 1656, et des réflexions sur les miracles. Le texte le plus ancien en pourrait être la pensée 922 à 924-753, fragment d'une méditation spirituelle qui développe exactement le même thème que la II lettre aux Roannez datée du 24 septembre 1656 ; P. Ernst y voit une note préparatoire, voire une première version de cette lettre. Quant au texte le plus tardif, le long et composite fragment 916 et 917-746, comportant encore de nombreuses références directes aux jésuites, il a sans doute été rédigé à la fin du mois d'octobre 1657, mais il s'agit sans doute d'une exception, l'immense majorité des fragments de cette strate étant contemporains des Provinciales, puisque Pascal utilise encore les grandes feuilles sur lesquelles il rédige ses « petites lettres ».

9 La strate suivante, nommée par P. Ernst «RC/DV », constitue en revanche une strate majeure, qui rassemble plus d'une centaine de fragments, et correspond à une période plus restreinte, s'étendant entre l'extrême fin de 1656 et mars 1657, donc contemporaine des XVII et XVIII ${ }^{e}$ lettres. L'utilisation d'un nouveau paquet de feuilles, de dimensions nettement inférieures à celles qu'utilisait Pascal pour les Provinciales, matérialise en quelque sorte la nouvelle orientation de l'auteur, qui délaissera progressivement la polémique au profit de l'apologie. Cependant, la plupart des fragments classés dans la liasse «Soumission et usage de la raison" prolongent encore la réflexion de la XVII ${ }^{e}$ Provinciale, datée du 23 janvier 1657, sur la souveraineté de l'intelligence confrontée à la tyrannie vaticane ${ }^{15}$. On observera que cette strate est chronologiquement « englobée " par la strate précédente qui, bien que ne représentant qu'un nombre restreint de fragments, s'étend sur un laps de temps beaucoup plus long: cette possible superposition complique l'appréhension de l'évolution du projet à ce stade. Toutefois, cette deuxième strate manifeste indéniablement une étape décisive du projet apologétique, que P. Ernst définit comme « l'émergence simultanée des thèmes majeurs ».

10 La troisième strate, majeure elle aussi, et nommée "strate au cor » par P. Ernst, est plus problématique : alors même que la période de rédaction à laquelle elle correspond (marsmai 1658, pour la plupart des fragments) ne recouvre pas l'époque des Provinciales et que 
la plupart des fragments qu'elle contient sont nettement postérieurs aux «petites lettres ", elle comprend plusieurs fragments manifestement polémiques, ou encore consacrés aux miracles. Le fragment 700-578, notamment, est manifestement lié à la campagne des Factums qui suit immédiatement les Provinciales. Exprimant l'indignation de Pascal devant la corruption et l'audace de la Compagnie en des termes proches de ceux du second Écrit pour les curés de Paris (rédigé tout entier le 2 avril 1658 ${ }^{16}$ ), il peut en effet être considéré comme un brouillon de ce texte.

11 Cependant, il présente également des ressemblances frappantes avec le Factum pour les curés de Paris, paru selon Hermant dès le 25 janvier $1658^{17}$, et pourrait donc être encore antérieur. Des analogies similaires suggèrent que certains des fragments rédigés sur cette rame pourraient bien être antérieurs à la période couverte par cette strate, autrement dit n'être que recopiés. Cette hypothèse est confirmée par des "papiers» tels que le fragment intitulé « Venise », dont P. Ernst situe la rédaction vers la mi-février 1657, c'està-dire à l'époque de la XVIII ${ }^{e}$ Provinciale. La rédaction initiale de certains fragments (très minoritaires) de cette strate peut donc remonter aux premiers mois de l'année 1657 : ainsi, les fragments « Prov. » (679-558) et « Montalte » (692-571) se réfèrent au recueil des dix-sept premières lettres, et ont donc sans doute été rédigés au cours du mois de février 1657, entre la XVII et la XVIII ${ }^{e}$ Provinciales. Quant aux fragments 379-411 et 648-533, ils constituent des réponses cinglantes au Rabat-joie des jansénistes publié en août 1656 et ont vraisemblablement été rédigés sur-le-champ par Pascal, c'est-à-dire vers septembre 1656 - même si leur transcription est plus tardive, et n'a sans doute été effectuée qu'au début de l'année suivante. D'autres études de détail permettraient de montrer que les fragments 724-605 à 728-609, 718-596 et 719-597, par exemple, sont contemporains de la XVIII Provinciale.

12 Néanmoins, la plupart des autres fragments de cette strate peuvent être datés de la fin du printemps 1658 , c'est-à-dire d'une époque nettement postérieure aux « petites lettres ${ }^{18}$ ». Ils sont certes contemporains des écrits connexes des Provinciales (Écrits des curés de Paris, Projet de mandement, etc.), mais nous ne les prendrons pas en compte, car ils ne datent pas de cette période cruciale où le projet des Provinciales proprement dites donne naissance au projet apologétique.

13 Quant aux autres strates mises au jour par P. Ernst, elles contiennent des fragments dont la date de rédaction ne coïncide plus avec l'époque des Provinciales.

\section{La première strate rédactionnelle}

14 La première strate relevée par P. Ernst, désignée par l'appellation "cadran B cœur C », contient quelques papiers qui seront regroupés dans le troisième dossier sur les miracles, écarté en juin 1658, et qui témoignent du lien le plus évident unissant les Pensées et les Provinciales : le miracle de la Sainte-Épine, à l'origine du projet apologétique de Pascal ${ }^{19}$ selon Gilberte :

Il lui vint à cette occasion de ce miracle particulier plusieurs pensées très importantes sur les miracles en général. [...] Comme toutes les vérités sont tirées les unes des autres, c'était assez qu'il se fût appliqué à une, les autres lui venaient comme à la foule [...]. Et ce fut en cette occasion qu'il se sentit tellement animé contre les athées que, voyant dans les lumières que Dieu lui avait données de quoi les convaincre et les confondre sans ressources, il s'appliqua à cet ouvrage ${ }^{20}$. 

aux Roannez rédigée vers le 29 octobre $1656^{22}$, c'est-à-dire à peu près contemporaine de la XIV Provinciale, est présente dans un autre papier de cette strate, non enregistré par la seconde copie mais appartenant au manuscrit Périer : 921-752. Or, ce thème, d'apparence toute apologétique, peut s'interpréter comme un prolongement du contexte polémique des Provinciales: de même que la vérité de la foi de Port-Royal et des miracles qui s'y étaient produits, à commencer par celui de la Sainte-Épine, demeurait cachée, de même «Jésus-Christ est demeuré inconnu parmi les hommes », comme l'Eucharistie demeure parmi le pain commun, «sans différence à l'extérieur ${ }^{23}$ ». Les Pensées élargissent cette perspective : qu'elle appartienne à l'ordre humain, scientifique, moral ou religieux, la vérité ne peut apparaître qu'au milieu des faussetés : de même que, sur le plan religieux, le miracle, par excellence signe divin manifeste, défend en vain la vérité aux yeux des hommes, de même - et a fortiori-les vérités d'ordre humain sont condamnées à demeurer ambivalentes, à la fois vraies et fausses, ce qui explique l'importance prise par le pyrrhonisme, dès cette strate, dans les Pensées. Ainsi le long fragment 903 à 905-450, appartenant aux notes sur les miracles de cette première strate, vise-t-il déjà indéniablement les libertins :

Pyrrhonisme

Chaque chose est ici vraie en partie, fausse en partie. La vérité essentielle n'est point ici, elle est toute pure et toute vraie. Ce mélange la déshonore et l'anéantit. Rien n'est purement vrai, et ainsi rien n'est vrai en l'entendant du pur vrai.

Pascal réintègre dans cette perspective, à titre d'exemple parmi d'autres, le problème de l'homicide, dont il avait débattu vigoureusement dans les Provinciales :

Rien n'est purement vrai [...]. On dira qu'il est vrai que l'homicide est mauvais. Oui, car nous connaissons bien le mal et le faux. Mais que dira-t-on qui soit bon ? [...] De ne point tuer? Non, car les désordres seraient horribles et les méchants tueraient tous les bons. De tuer? Non, car cela détruit la nature. Nous n'avons ni bien ni vrai qu'en partie, et mêlés de mal et de faux.

D'un côté, le meurtre représente par excellence le mal que la loi naturelle inscrite en chaque homme lui permet de connaître pour tel. Ce savoir, bien que purement négatif, n'est pas nul : c'est sur lui que se fondait l'auteur des Provinciales. De l'autre, Pascal, qui n'est plus tenu ici aux strictes nécessités de la polémique contre les jésuites, peut désormais exploiter l'argument inverse : le droit de tuer est nécessaire pour préserver l'ordre social des individus qui cherchent à l'anéantir ${ }^{24}$. Ces arguments ne sont pas contradictoires, dans la mesure où ce droit de tuer est réservé à la collectivité, représentée par les juges. Cependant, si la XIV ${ }^{\mathrm{e}}$ Provinciale, légèrement postérieure à ce fragment selon $\mathrm{T}$. Shiokawa ${ }^{25}$, les représentait comme scrupuleux et soucieux de s'entourer de toutes les précautions nécessaires pour éviter l'injustice, les Pensées en

Courrier du Centre international Blaise Pascal, 25 | 2015 
donnent une image beaucoup plus satirique : jouet ridicule de l'imagination, fantoche dérisoire, le juge y constitue une figure emblématique du scepticisme généralisé qui doit dans un premier temps déstabiliser le lecteur de l'Apologie ${ }^{26}$.

Or, le fragment «Pyrrhonisme» cité ci-dessus, voisinant avec des notes directement relatives aux miracles et aux jésuites, pourrait bien, comme le suggère $T$. Ishikawa ${ }^{27}$, ne pas viser uniquement les vérités d'ordre humain, mais s'appliquer également aux vérités religieuses : la même ambivalence caractérise en effet pour Pascal les valeurs morales et des réalités religieuses telles que le miracle bien sûr, mais aussi l'Eucharistie, la doctrine de la grâce, l'Église même. Si le problème de l'existence de la loi naturelle est au cœur du rapport (rupture ou continuité) entre les Provinciales et les Pensées ${ }^{28}$, c'est que, plus largement, le vrai, le bien, dans le domaine sacré comme dans le domaine profane, sont condamnés à n'apparaître ici-bas que mêlés au faux et au mal, "sans différence à l'extérieur ». Pascal généralise dont ici la réflexion des Provinciales en prenant acte, au cours de sa réflexion sur le miracle et la morale jésuite, de l'« isomorphie ${ }^{29}$ » de la vérité dans l'ordre religieux et dans l'ordre humain. Or, ce rapport équivoque à la vérité étant commun à tous les hommes, cette isomorphie est également celle des conditions respectives du croyant et de l'athée, et favorise par-là la sympathie (au sens étymologique du terme) du second pour le premier, précieux appui du projet apologétique. Elle permet ainsi le passage harmonieux de la polémique interne au catholicisme à l'apologie, tournée aussi vers l'incroyant, qui, ressentant lui-même ce clair-obscur généralisé dans l'ordre purement humain, pourra facilement comprendre l'équivocité intrinsèque de l'état de foi.

21 Surtout, simultanément et conjointement au thème du « Dieu caché » apparaît un autre grand thème apologétique : la théorie des trois états. Étroitement liée au thème du Deus absconditus, il n'est guère surprenant qu'elle s'élabore à peu près à la même époque, et que sa formulation définitive corresponde au moment - éminemment critique, dans tous les sens du terme - où sont rédigées simultanément les dernières Provinciales et les premières notes destinées à l'Apologie. Cette triade est explicitée pour la première fois dans la XVI ${ }^{e}$ Provinciale $e^{30}$, où elle apparaît d'emblée associée aux thèmes de la figure d'une part, de l'Eucharistie d'autre part ${ }^{31}$ (il s'agit en effet de défendre la Fréquente Communion ainsi que la Seconde lettre à un duc et pair de France, ouvrages accusés d'hérésie calviniste par les jésuites) :

L'état des Chrétiens [...] tient le milieu entre l'état des bienheureux et l'état des Juifs. Les bienheureux possèdent Jésus-Christ réellement sans figure et sans voile. Les Juifs n'ont possédé de Jésus-Christ que les figures et les voiles, comme était la manne et l'agneau pascal. Et les Chrétiens possèdent Jésus-Christ dans l'Eucharistie véritablement et réellement, mais encore couvert de voiles. ${ }^{32}$

C'est donc à l'occasion de la dispute sur l'Eucharistie que Pascal formule d'abord cette théorie : si «l'Eucharistie est parfaitement proportionnée à notre état de foi ", c'est parce que celui-ci possède un statut foncièrement équivoque et ambivalent, milieu entre celui des Juifs et celui des bienheureux. En réfléchissant sur les miracles, second catalyseur du projet apologétique, Pascal avait pu également méditer sur l'aveuglement des Juifs, qu'il mentionne à de nombreuses reprises dans les trois dossiers sur les miracles. Il en tire cette conclusion que la vérité étant par nature une et immuable, c'est sa manière d'être qui varie avec le temps, entraînant la théorie des trois états. D'où l'affinité de cette dernière avec un autre thème pascalien majeur, celui du Dieu caché : plus l'homme monte d'état en état, de bas en haut, plus Dieu se découvre à lui ${ }^{33}$. Au cours du second stade de l'histoire, l'homme se tient en un mi-lieu, instable sinon improbable; plusieurs fragments de cette première phase rédactionnelle, tous issus du même demi-feuillet que la 
remarque ci-dessus, développent cette idée directement issue de la théorie des trois états :

La grandeur de l'âme humaine consiste à savoir s'y tenir. Tant s'en faut que la grandeur soit à en sortir qu'elle est à n'en point sortir ${ }^{34}$.

C'est sortir de l'humanité que de sortir du milieu ${ }^{35}$.

La nature nous a si bien mis au milieu que si nous changeons un côté de la balance,

nous changeons aussi l'autre ${ }^{36}$.

L'état des chrétiens est intermédiaire entre celui, antérieur et inférieur, des Juifs, vivant dans un monde purement figuratif, et celui, postérieur et supérieur, des bienheureux, habitant la pure vérité37.

En lien direct avec cette tripartition apparaît dès les Provinciales une structure de pensée qui sera récurrente dans les Pensées: la définition de la vraie foi par opposition à deux erreurs contraires et symétriques. En effet, comme le soulignent la IXe et surtout la XVI ${ }^{e}$ Provinciales, la bonne compréhension du mystère de l'Eucharistie exige le rejet de deux erreurs contraires : celle des protestants, et celle des jésuites. Ce mode d'approche de la vérité, qui n'est pas sans rappeler la notion trop souvent simplifiée du «juste milieu » aristotélicien ${ }^{38}$, réapparaîtra à plusieurs reprises dans les Pensées, par exemple à propos des deux grandes philosophies rationnelles (stoïcisme et pyrrhonisme) définissant les deux pôles opposés et mensongers par rapport auxquels se détermine la vérité chrétienne en sa médiété. Par ce témoignage de l'équivocité foncière, non seulement de la vérité icibas, mais aussi de l'état des chrétiens, mi-lieu entre deux autres états, commence à se préciser le lien entre la partie théologique et la partie proprement anthropologique de la future Apologie.

Et c'est bien parce que la vérité ici-bas ne peut échapper à l'ambiguïté, à l'opacité, que la recherche de critères pour la définir est condamnée à être à la fois nécessaire et insuffisante. Ce statut problématique de la règle, au centre des Provinciales à travers la mise en question de la casuistique, sera réutilisé dans la stratégie déstabilisatrice de l' Apologie. Le fragment 601-498, encore très lié à la polémique anti-jésuite, est significatif de ce glissement :

Les casuistes soumettent la décision à la raison corrompue et le choix des décisions

à la volonté corrompue, afin que tout ce qu'il y a de corrompu dans la nature de

l'homme eût part à sa conduite.

En érigeant abusivement la fantaisie, le "bon vouloir " (l'épithète ressemble fort à une ironie du sort linguistique) en créateurs de règles, la casuistique menace jusqu'à la loi qu'elle prétendait faire triompher. Sur un autre plan, les Pensées exploiteront cette fragilité des lois et des repères humains dans le cadre d'un vacillement pyrrhonien organisé, première étape de la conversion.

27 La critique des « fantaisies » jésuites, cette flatterie d'une nature humaine corrompue, est également au fondement de la méditation pascalienne sur le divertissement. Elle apparaitt en filigrane dès la IV ${ }^{e}$ Provinciale, lorsque Pascal accuse les bons Pères de pousser l'âme à se détourner de la pensée de Dieu à travers la direction d'intention :

Quand on a pu gagner une foi sur soi de n'y plus penser du tout, toutes choses

deviennent pures pour l'avenir ${ }^{39}$.

Dans les Pensées, cet argument ne trouvera son plein développement que dans l'étape rédactionnelle suivante, mais dès cette première strate, le fragment 79-114 amorce cette thématique : cet homme qui préfère à son épouse légitime, avec laquelle il vivait heureux, l'entreprise ludique de séduction d'une autre femme, est à l'image de tous ses semblables, 
qui recherchent « la chasse plutôt que la prise ${ }^{40}$ ». Son ennui lorsqu'il se replonge dans ce qui faisait autrefois son bonheur met en lumière la vanité de tout contentement aux fondements purement humains. Surtout, le fragment 166-198, aux résonances déjà pleinement apologétiques, souligne l'urgence de ne plus se contenter du divertissement :

Nous courons sans souci dans le précipice après que nous avons mis quelque chose devant nous pour nous empêcher de le voir.

C'est que le thème du divertissement fournit à l'apologiste un argument décisif : la nécessité urgente de se « convertir », de faire face aux questions essentielles, que souligne également le fragment 164-196:

Je trouve bon qu'on n'approfondisse pas l'opinion de Copernic, mais ceci.

Il importe à toute la vie de savoir si lâme est mortelle ou immortelle.

La référence à Copernic est particulièrement significative : l'astronome était en effet également mentionné dans la XVIII ${ }^{e}$ Provinciale, mais dans une toute autre perspective, puisqu'il s'agissait alors de montrer les limites de l'autorité du pape en défendant l'auteur du De Revolutionibus. Au contraire, dans les Pensées, le savant polonais représente la vanité des recherches cosmologiques opposée à l'importance essentielle de la métaphysique.

31 La Compagnie ne fournit pas seulement à Pascal un exemple frappant de légitimation du divertissement; son usage de la calomnie entraîne l'apologiste dans une réflexion qui dépassera celle menée dans les Provinciales. En effet, si celles-ci attribuaient essentiellement les calomnies jésuites à une maladresse polémique, D. Descotes montre qu'un «papier» tel que le fragment 962-796, en apparence composé de notes préparatoires à la XVe lettre (datée du 25 novembre 165641) approfondit en fait considérablement le problème posé par cette Provinciale, en attribuant la calomnie à un aveuglement aussi «surnaturel ${ }^{42}$ " que dénaturé, et voué à s'autodétruire. L'« avanttexte » fourni par les Pensées, loin de se réduire à un simple brouillon, suggère donc ici une interprétation plus complète et plus profonde, qui sera étendue à d'autres contremodèles dans l'Apologie, en particulier les philosophies stoïcienne et pyrrhonienne, qui, si elles ne s'autodétruisent pas à proprement parler, se détruisent du moins mutuellement, suggérant ainsi l'autodestruction de la philosophie rationnelle ${ }^{43}$. Le thème de la calomnie témoigne ainsi de la manière dont « Pascal sait prendre à son compte une notion que les circonstances lui imposent pour en tirer un concept-clé dont la portée est philosophique et même religieuse, et la matière d'une réflexion qui conduit aux larges perspectives des Pensées ${ }^{44} »$.

Parallèlement à cette dénonciation du divertissement et de l'aveuglement, c'est naturellement le pouvoir de la raison qui est mis en cause. Les Provinciales n'hésitent pas, on le sait, à appuyer leur argumentation sur le bon sens du lecteur, au point qu'on a pu les interpréter comme le courageux manifeste de l'esprit d'examen, défenseur de Galilée luimême, en face d'une tyrannie spirituelle. Dans les Pensées, au contraire, Pascal serait gagné par un fanatisme (ou du moins un pessimisme) croissant, et ne donnerait plus d'autre conseil à la raison que celui de "s'abêtir "; de fait, dès les premiers fragments de l' Apologie, contemporains des "petites lettres ", Pascal s'interroge sur l'impuissance de la raison. L'opposition entre les deux œuvres à ce sujet demeure donc hautement problématique, même si la chronologie rend une rupture radicale des plus invraisemblables. Voici en effet ce qu'écrit encore Pascal dans la XVIII Provinciale :

D'où apprendrons-nous donc la vérité des faits? Ce sera des yeux, mon Père, qui en sont les légitimes juges, comme la raison l'est des choses naturelles et intelligibles, et la foi des choses surnaturelles et révélées. [...] ces trois principes de nos 
connaissances, les sens, la raison et la foi, ont chacun leurs objets séparés, et leur certitude dans cette étendue ${ }^{45}$. fragments contemporains des Provinciales, rédigés entre fin 1656 et mars 1657, comporte un seul "papier» appartenant de manière certaine à cette première phase rédactionnelle: le fragment 188-220, qui peut d'ailleurs être considéré comme le plus ancien de cette liasse ${ }^{46}$, et qui, incontestablement, relève plus de la «soumission » que de l'« usage » de la raison:

La dernière démarche de la raison est de reconnaître qu'il y a une infinité de choses qui la surpassent. Elle n'est que faible si elle ne va jusqu'à connaître cela.

Que si les choses naturelles la surpassent, que dira-t-on des surnaturelles?

Au moment même où il invoque les droits du bon sens et de la raison dans la polémique qui l'oppose aux casuistes ${ }^{47}$, Pascal réfléchit donc sur les limites de cette même raison. Il conservera ce fragment dans la liasse "Soumission et usage de la raison", et le contrebalancera ensuite par des fragments de la strate suivante ( $«$ Excellence de cette manière de prouver Dieu ») affirmant la puissance de la raison. Cet apparent paradoxe suggère que dès cette première strate rédactionnelle, le projet apologétique était relativement avancé dans l'esprit de Pascal : c'est bien en effet sur le terrain de l'athée, de celui qui «n'admet que la raison », que se placera l'apologiste en montrant que la raison elle-même implique un désaveu de la raison.

C'est ainsi que, dès la première étape du projet apologétique, pourtant pleinement contemporaine des Provinciales, Pascal amorce le vacillement salutaire qu'il généralisera dans son Apologie. Il s'agit - dans un premier temps du moins - de jeter le trouble, de refuser les règles trop faciles des casuistes :

On aime la sûreté, on aime que le pape soit infaillible en la foi, et que les docteurs graves le soient dans les mœurs, afin d'avoir son assurance ${ }^{48}$.

Or, précisément, le chrétien est celui qui sait refuser cette fausse tranquillité, vivre pleinement le risque continuel et foncier de la condition humaine, et en particulier de la condition chrétienne, et ne se reposer qu'en Dieu. Les Pensées correspondent donc à cet égard à un infléchissement non négligeable de la pensée pascalienne.

Plusieurs thèmes apologétiques capitaux font donc leur apparition dès cette toute première étape du projet apologétique, en lien plus ou moins direct avec la querelle des Provinciales. Il n'est pas jusqu'à l'ethos - ô combien problématique, on le sait ${ }^{49}-\mathrm{de}$ l'auteur des Provinciales qui ne sera réinvesti dans la perspective apologétique: un fragment appartenant à la première strate rédactionnelle, et se référant manifestement à l'auteur des " petites lettres » proclamant qu'il fustige les jésuites pour leur propre bien, et sera tout prêt à les accueillir en frères dès qu'il seront convertis, sera classé dans la liasse intitulée "Morale chrétienne»: «Il aime ses proches, mais sa charité ne se renferme pas dans ces bornes et se répand sur ses ennemis, et puis sur ceux de Dieu » (355-387). C'est que la première strate rédactionnelle est déjà postérieure au changement de stratégie de Pascal dans ses "petites lettres » : accusé de tourner en dérision les choses saintes, il s'en justifie dans la $\mathrm{XI}^{\mathrm{e}}$ Provinciale, mais, sans doute sensible au reproche, abandonne l'ironie dans les lettres suivantes ${ }^{50}$. 


\section{La deuxième strate rédactionnelle}

Cest pourtant la deuxième strate rédactionnelle mise en lumière par P. Ernst, contemporaine des $\mathrm{XVII}^{\mathrm{e}}$ et XVIII ${ }^{e}$ Provinciales, qui voit la naissance quasi simultanée des thématiques majeures de l'Apologie. Les définitions essentielles sont posées: la "conversion véritable", la vraie vertu, et même la vraie religion et les critères qui permettent de la reconnaitre et de l'opposer aux fausses croyances aussi bien qu'aux mauvais croyants, représentés à l'époque de la rédaction par les jésuites. Surtout, tous les grands thèmes sont présents, qu'ils soient directement liés à la thématique matricielle du Deus absconditus (le double sens, la théorie des figuratifs, l'opposition entre hommes charnels et spirituels) ou qu'ils entretiennent avec elle un lien plus lâche (la perpétuité, l'usage et la soumission de la raison, etc.). En revanche, dès cette deuxième phase, l'argument du miracle - qu'on avait pu prendre pour le fondement premier de l'Apologie est écarté, «éliminé sans appel, sans équivoque aucune, vigoureusement et de façon définitive ${ }^{51} »$ des futurs fragments classés. Il ne subsiste, de manière indirecte, que sous la forme de développements concernant la théorie du Dieu caché, et en conséquence la nécessité d'une herméneutique et surtout l'accomplissement des prophéties, «miracle subsistant ». Cette strate correspond donc incontestablement à une étape capitale et singulière du projet apologétique, même si P. Ernst souligne que, comme la précédente, elle se caractérise encore par une nette prédominance de l'approche théologique de la condition humaine; la perspective proprement anthropologique de l'Apologie ne sera développée qu'ultérieurement, et sans doute l'importance qu'elle prendra, tout à fait exceptionnelle dans la tradition apologétique, n'est-elle pas encore pleinement pressentie par Pascal.

Dans ce qui deviendra la liasse "Fondements de la religion et réponses aux objections », centrée sur le thème du Dieu caché, Pascal semble laisser de côté, à ce stade de la rédaction, le lien avec l'Eucharistie qu'il développait dans la strate précédente, pour insister plutôt sur le fait que Dieu aveugle certains hommes, tels les jésuites refusant de voir dans les miracles produits à Port-Royal une marque d'approbation divine :

On n'entend rien aux ouvrages de Dieu si on ne prend pour principe qu'il a voulu aveugler les uns et éclaircir les autres ${ }^{52}$.

Quant au fragment 158-190, qui sera rangé dans la liasse « Commencement», il associe au thème du Dieu caché celui, proprement apologétique on l'a vu, de la nécessité urgente de sa recherche.

41 Surtout, le thème du Deus absconditus donne naissance à une question centrale dans l' Apologie, celle de l'interprétation. C'est que l'ambiguïté fondamentale de toute vérité icibas est liée à l'existence de vérités figuratives, à la fois vraies et fausses. Or, dans la liasse intitulée "Que la loi était figurative», Pascal propose une réflexion herméneutique qui lui a sans nul doute été inspirée par les controverses avec les jésuites, ainsi que par la polémique liées aux miracles de Port-Royal. En effet, comme le remarque P. Force ${ }^{53}$, lorsqu'ils cherchent à réduire les contradictions existant entre leur doctrine et l'Écriture, les jésuites adoptent une démarche exactement contraire à l'herméneutique que prônent les Provinciales et les Pensées:

Soit par l'interprétation des termes, soit par la remarque des circonstances favorables, soit enfin par la double probabilité du pour au contre, on accorde toujours ces contradictions prétendues ${ }^{54}$. 
« circonstances favorables » recouvrent en fait un attachement de mauvaise foi à la lettre du texte, si « la double probabilité du pour au contre » oppose de manière illégitime à la doctrine de la tradition celle d'un particulier (ou d'un groupe de particuliers), "l'interprétation des termes » en revanche, c'est-à-dire l'attribution d'un sens figuré ou dérivé à un terme de l'Écriture, est une pratique courante et parfaitement admise par Pascal. Si l'herméneutique jésuite est sacrilège, ce n'est pas parce qu'elle utilise cette méthode, c'est parce qu'elle la met au service d'une morale relâchée ${ }^{55}$. En outre, en allant parfois jusqu'à attribuer deux sens contradictoires à l'Écriture, elle viole le principe fondamental de cohérence exposé dès cette seconde strate rédactionnelle :

Tout auteur a un sens auquel tous les passages contraires s'accordent ou il n'a point de sens du tout ${ }^{56}$. 位 critères de croyance requis par le christianisme, et à ce titre risque d'agir comme contretémoignage, d'éloigner encore les hommes de la vraie foi.

Au contraire, Montalte se livre pour sa part à un scrupuleux travail d'interprétation de la doctrine jésuite en cherchant la raison d'effets apparemment contradictoires, tels que la coexistence chez les jésuites de confesseurs sévères et relâchés. La Compagnie faisant corps avec tous ses représentants, c'est-à-dire se donnant en quelque sorte pour un auteur unique, l'enquêteur des Provinciales a toute légitimité pour établir des rapprochements systématiques entre divers points de la doctrine jésuite.

Est-ce à dire que le problème de l'interprétation de l'Écriture, central dans les deux œuvres, y soit abordé sous le même angle ? Il n'en est rien, et pour des raisons évidentes. Dans les Provinciales en effet, Pascal s'adresse à des catholiques pour qui la parole divine est infaillible : la nécessité herméneutique est fondée sur la présupposition de la vérité du texte sacré. Il distinguait donc d'une part une herméneutique sacrée, d'autre part une herméneutique profane, qui fondait sur le raisonnement et sur le rapport des sens "l'intelligence du sens d'un auteur " (qu'il s'agisse de Jansénius ou des nouveaux casuistes) $^{57}$. Dans les Pensées au contraire, s'adressant à des incroyants, Pascal, d'un simple point de vue argumentatif ${ }^{58}$, ne peut partir de ce postulat et doit faire reposer la nécessité d'interpréter le texte sur la simple hypothèse de sa cohérence : il applique à l'Écriture une herméneutique qui ne présuppose nullement la vérité de la Révélation, mais cherche simplement à élucider empiriquement le sens d'un texte donné. L'expression «sens d'un auteur " peut alors désigner la compréhension des prophètes eux-mêmes, qui autorisent tout à fait une lecture "profane », selon des principes "naturels et clairs ${ }^{59}$ ", pour les croyants comme pour les incroyants : comme les auteurs profanes des "petites lettres", les prophètes autorisent une lecture « objective », leur intelligence peut être qualifiée de «point de fait ${ }^{60} »$.

Plus largement, deux domaines d'exercice de la raison bien distincts sont abordés dans Les Provinciales : l'exégèse, où le raisonnement a un rôle précis à jouer, et la superstition, qui manifeste une démission abusive de la raison. Ces thèmes seront repris sous une forme nettement infléchie dans les Pensées : le problème général de l'exégèse fera place à l'établissement de la vérité des Écritures par un argument particulier, la preuve prophétique, et - c'est le point qui nous intéresse ici, car la preuve prophétique n'est pas encore pleinement mise en place à l'époque des Provinciales - la critique de la superstition s'élargira en une méditation plus nuancée sur l'usage et surtout la soumission de la raison dans la religion véritable.

Courrier du Centre international Blaise Pascal, 25 | 2015 
47 Le passage du contexte polémique à l'exploitation apologétique se fait par un double processus d'inversion (de symétrie) et de généralisation. Le fragment 808-655, qui présente la religion chrétienne comme la seule qui combine « la raison, la coutume et l'inspiration ", constitue un élargissement a contrario, de tout le dispositif argumentatif de la IV Provinciale: en opposant au jésuite non seulement l'honnête homme qu'est Montalte, s'appuyant sur l'expérience et le bon sens, mais aussi son ami, fort de son érudition philosophique et théologique, Pascal suggérait que le laxisme jésuite était à la fois condamné par la raison naturelle et par la tradition chrétienne. Cette dernière garantie est développée dans les Pensées par le fragment 285-317, qui donne toute sa portée au respect de la tradition que manifestait l'ami de Montalte :

Si l'ancienne Église était dans l'erreur, l'Église est tombée. Quand elle y serait aujourd'hui, ce n'est pas de même, car elle a toujours la maxime supérieure de la tradition de la créance de l'ancienne Église. Et ainsi cette soumission et cette conformité à l'ancienne Église prévaut et corrige tout. Mais l'ancienne Église ne supposait pas l'Église future et ne la regardait pas, comme nous supposons et regardons l'ancienne.

Le contexte polémique de la rédaction de ce fragment permet certes de supposer que ce sont notamment les abus des nouveaux casuistes qui rendent ce rempart plus nécessaire que jamais, mais sa présence au sein des liasses classées (liasse «Perpétuité ») permet d'affirmer que Pascal souhaitait intégrer cet argument dans son Apologie.

Plus largement, le fragment 808-655 constitue une rigoureuse inversion du contre-modèle jésuite. Dans les Provinciales, Pascal reproche en effet à la Compagnie de vouloir imposer au public de "croire que des propositions sont dans un livre, quoiqu'on ne les voie pas ", alors même que, comme l'affirme la XVIII lettre, « la nature des choses de fait » est de ne se prouver «que par les sens ${ }^{61}$ ». Surtout, il accuse les jésuites de pêcher à la fois contre la raison - Montalte ne cesse d'en appeler contre eux au sens commun -, contre la coutume véritable - les «nouveaux casuistes » ne fabriquent une fausse tradition que pour mieux utiliser le processus de la coutume à leur profit ${ }^{62}$-, et contre la grâce, "l'inspiration » puisqu'ils croient pouvoir se dispenser de l'amour de Dieu. C'est par contraste avec la foi dépravée des jésuites, instrumentalisée au service de leur volonté de puissance, que Pascal, dans son Apologie, définira la véritable foi chrétienne. Celle-ci met au service de la vérité les trois critères fondamentaux de la croyance, en particulier «l'inspiration ", le cœur, étranger aux (pseudo-) démonstrations interminables des casuistes : « La foi est un don de Dieu, ne croyez pas que nous disions que c'est un don de raisonnement ${ }^{63}$ ", affirme Pascal dès cette époque. Il va même désormais jusqu'à définir la vraie religion comme celle qui s'oppose à la raison, prenant alors pleinement le contre-pied des Provinciales :

La seule religion contre la nature, contre le sens commun, contre nos plaisirs, est la seule qui ait toujours été ${ }^{4}$.

50 Cette réorientation capitale, amorcée, on l'a vu, dès la première strate, est confirmée par le Premier Écrit des Curés de Paris, daté du 25 janvier 1658, qui reproche aux jésuites leur confiance abusive et impie en la « seule raison naturelle ${ }^{65}$ ».

51 Le rôle que Pascal attribuait à la raison à cette époque charnière apparaît donc comme hautement problématique. La liasse qui sera intitulée « Soumission et usage de la raison, en quoi consiste le vrai christianisme" fournit pour son élucidation de précieux renseignements, dans la mesure où, de manière significative, elle réunit essentiellement des fragments rédigés entre fin 1656 et mars 1657, c'est-à-dire à l'époque de la XVII ${ }^{\mathrm{e}}$ Provinciale. Encore tout proches des polémiques sur les miracles, de nombreux «papiers » 
de cette liasse comptent donc parmi les plus anciens du projet d'Apologie, qu'ils appartiennent à la première strate (c'est par exemple le fragment 188-220 cité plus haut) ou à la deuxième : c'est notamment le cas des fragments 179-210 et 181-212, condamnant la superstition, fruit d'une soumission excessive de la raison. Le lien avec la polémique des Provinciales n'est pas explicite, mais il est possible de le reconstituer, comme l'a montré Ph. Sellier ${ }^{66}$ : c'est en effet d'abord face aux coups de force de Rome que Pascal défend la royauté de la raison. Ainsi, comme la plupart des fragments qui seront classés dans cette liasse, le fragment 181-212:

La piété est différente de la superstition.

Soutenir la piété jusqu'à la superstition, c'est la détruire. [...]

Impiété de ne pas croire l'Eucharistie sur ce qu'on ne la voit pas.

Superstition de croire des propositions, etc.,

prolonge la réflexion de la XVII ${ }^{e}$ Provinciale (qui coïncide avec le passage de la première à la deuxième strate rédactionnelle des Pensées) sur le rôle que doit jouer l'intelligence face à la tyrannie romaine, et annonce la thématique de la lettre suivante : le rôle du libreexamen en matière de fait. Pascal y définit là encore le bon usage de la raison que suppose la vraie religion comme un milieu entre la remise en cause généralisée des protestants, qui soumettent leur foi en l'Eucharistie aux sens purement humains, et la soumission aveugle au pape en des matières qui n'engagent nullement son autorité ${ }^{67}$. Ce fragment se rattache donc de toute évidence au contexte polémique, tout en en élargissant déjà la portée dans une perspective apologétique, comme en témoigne notamment la prise en compte de l'image donnée aux « hérétiques » et sa présence dans les liasses classées.

Il est d'ailleurs hautement significatif que, selon P. Ernst, il ait été initialement rédigé, non seulement sur le même feuillet, mais directement à la suite du fragment 604-501, qui dénonce explicitement la tyrannie (en l'occurrence, vaticane) :

Église, pape.

Unité / multitude.

[...] La multitude qui ne se réduit point à l'unité est confusion.

L'unité qui ne dépend pas de la multitude est tyrannie.

Il n'y a presque plus que la France où il soit permis de dire que le concile est audessus du Pape.

Le contexte est manifestement le même que celui qui a présidé à la rédaction du fragment 567-473, appartenant à la même strate, qui lie à ce thème celui du milieu, de l'opposition à deux erreurs opposées et complémentaires :

L'unité et la multitude: Duo aut tres in unum. Erreur à exclure l'un des deux, comme font les papistes, qui excluent la multitude, ou les huguenots, qui excluent l'unité.

Comme le suggère déjà le fragment 181-212 cité plus haut, le concept de tyrannie est en outre rattaché à la distinction des "ordres »: obliger des théologiens à approuver des textes ambigus consiste à user de la force au royaume des esprits, c'est-à-dire hors de son ordre ; ce sera la définition même de la tyrannie, concept apologétique qui possède donc une origine polémique, et plus précisément anti-papale ${ }^{68}$. Or, la deuxième strate rédactionnelle comporte au moins sept fragments se rapportant, de près ou de loin, à ce thème, notamment les fragments dénonçant la superstition, crédulité selon la chair (206-175, 207-176, 208-177, 210-179) et les fragments sur Venise, qui soulignent la nature proprement « politique » de la Compagnie ${ }^{69}$.

Dès cette époque, Pascal dénonce donc simultanément deux positions contraires et également déraisonnables : celle des jésuites d'une part, qui «manque[nt] de savoir où il 
faut juger ", et celle des incrédules ou des hérétiques d'autre part, qui "manque[nt] de savoir où il faut se soumettre ». Leur mise en rapport dans les Pensées s'explique par le constat, datant de la fin des Provinciales, que ces deux erreurs s'engendrent l'une l'autre : l'excès de soumission des uns détourne les autres de se soumettre là où il le faudrait, et donne prétexte aux adversaires de la vraie foi, comme Pascal le remarque dès la XVIII ${ }^{e}$ Provinciale, manifestant par là dès cette époque un souci proprement apologétique :

Ce serait [...] le moyen de fermer l'entrée [de notre religion] aux hérétiques et de leur rendre l'autorité du pape méprisable, que de refuser de tenir pour catholiques ceux qui ne croiraient pas que des paroles sont dans un livre où elles ne se trouvent point, parce qu'un pape l'aurait déclaré par surprise ${ }^{70}$.

Choquer les principes de la raison en un domaine où elle possède une légitimité ne peut qu'aboutir à rendre la religion « absurde et ridicule ${ }^{71} »$. Là encore, l'entrée dans l'apologie se fait par la transformation du lien de cause à conséquence en un lien de symétrie, mettant en valeur l'« entre-deux » que représente l'état du chrétien véritable - ce sera le thème du milieu. L'erreur des jésuites, excluant la raison et tombant de ce fait dans la superstition, aide à penser l'erreur analogue et inverse des libertins, n'admettant que la raison et tombant de ce fait dans le scepticisme :

Il y a peu de vrais chrétiens. Je dis même pour la foi. Il y en a bien qui croient, mais par superstition. Il y en a bien qui ne croient pas, mais par libertinage. Peu sont entre-deux ${ }^{72}$.

Plus grave encore : ceux même qui s'abandonnent à ces mauvais penchants manifestent par là leur incapacité à distinguer la véritable nature des choses :

Quelque proposition qu'on nous présente à examiner, il faut d'abord en reconnaître

la nature, pour voir auquel des trois principes nous devons nous en rapporter ${ }^{73}$.

De même que les jésuites méconnaissent la nature des «choses qui tombent sous les sens », erreurs dont témoigne leur manière de considérer les « formalités » :

C'est être superstitieux de mettre son espérance dans les formalités, mais c'est être superbe de ne vouloir s'y soumettre ${ }^{74}$,

les libertins ignorent la nature de la foi. D’après P. Ernst, ce " papier » a été rédigé avant les fragments 856 à 858-436 et 437, très vraisemblablement destinés à un écrit polémique sur les miracles, peut-être la fameuse « Provinciale sur les miracles » qui, selon H. Gouhier, aurait été commencée vers février 1657, puis rapidement abandonnée ${ }^{75}$. Ce raboutage prouve que dès cette époque, Pascal, dans un fragment manifestement polémique, distinguait très nettement les "formalités " de la religion véritable; il semble avoir rapidement compris le parti apologétique qu'il pouvait tirer d'une telle distinction.

Le raisonnement par symétrie ne se limite pas à la présentation du libertin comme inverse et analogue du jésuite : il semble avoir joué un rôle essentiel dans l'élaboration de la stratégie apologétique à partir de l'argumentaire polémique. C'est en effet à partir de cette exclusion de deux erreurs contraires que Pascal élabore dès cette deuxième strate rédactionnelle, comme en négatif, un argument apologétique absolument neuf: la présentation du christianisme comme union des vérités contraires. Le vrai chrétien est celui qui sait éviter à la fois deux excès, et concilier les droits de la raison et les droits de la foi selon les principes exposés dans le Fragment d'un traité du vide :

Les deux raisons contraires. Il faut commencer par-là : sans cela, on n'entend rien, et tout est hérétique. Et même, à la fin de chaque vérité, il faut ajouter qu'on se souvient de la vérité contraire ${ }^{76}$.

60 Plus généralement, le problème jésuite, qui constituait dans les Provinciales le fondement même de la discussion est en quelque sorte ravalé au rang de simple exemple, de base 
référentielle non essentielle. Le cas particulier de l'orgueil de la Compagnie, stigmatisé dans les "petites lettres» se trouve conceptualisé dans les Pensées afin de servir à une démonstration non seulement plus large, mais aussi profondément remaniée. Ainsi, l'audace prométhéenne des jésuites, trop prompts à s'affranchir de la lettre du texte sacré, donne naissance dans l'Apologie à une critique plus générale des «superbes", des « habiles » : la Compagnie, qui n'est plus explicitement mentionnée, est par là érigée au rang de contre-modèle archétypal et universel :

La seule religion chrétienne est proportionnée à tous, étant mêlée d'extérieur et d'intérieur. Elle élève le peuple à l'intérieur, et abaisse les superbes à l'extérieur, et n'est pas parfaite sans les deux, car il faut que le peuple entende l'esprit de la lettre et que les habiles soumettent leur esprit à la lettre ${ }^{77}$.

61 Surtout, l'assimilation des casuistes aux Juifs charnels effectuée dès cette époque donne naissance, par systématisation et généralisation, à une «théorie des deux états »structurant l'histoire religieuse de l'humanité entière. De même que la $\mathrm{X}^{\mathrm{e}}$ Provinciale accusait les jésuites de « décharger les hommes de l'obligation pénible d'aimer Dieu actuellement ", de même le fragment 286-318 porte :

Deux sortes d'hommes en chaque religion

Parmi les païens, des adorateurs de bêtes, et les autres adorateurs d'un seul dieu dans la religion naturelle.

Parmi les juifs, les charnels et les spirituels, qui étaient les chrétiens de la loi ancienne.

Parmi les chrétiens, les grossiers, qui sont les Juifs de la Loi nouvelle.

Les Juifs charnels attendaient un Messie charnel et les chrétiens grossiers croient que le Messie les a dispensés d'aimer $\operatorname{Dieu}^{78}$.

Les jésuites, qui se sont eux même appelés "Pharisiens de la loi nouvelle ${ }^{79}$ ", ne constituent plus que l'illustration la plus évidente de ces « chrétiens grossiers » croyant ployer Dieu à leurs désirs par la répétition mécanique de rituels privés de leur sens. Dans le Projet de mandement de $1657^{80}$, Pascal comparera de même les casuistes aux «faux

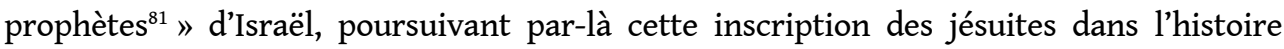
religieuse universelle; a contrario, la véritable foi consiste à leur faire obstacle, de même que les « vrais juifs » se sont opposés aux faux prophètes ${ }^{82}$. L'exemple aussi circonstanciel que matriciel des Provinciales a donc permis à l'apologiste de construire la catégorie conceptuelle des hommes "charnels", et d'étendre sa démarche herméneutique à l'histoire religieuse universelle.

Or, selon P. Ernst, ce même fragment 286-318 était à l'origine immédiatement suivi du fragment 365-397, manifestement rédigé en plein contexte polémique, lors de la querelle concernant la signature du premier Formulaire (cliché A63) :

L'expérience nous fait voir une différence énorme entre la dévotion et la bonté. 


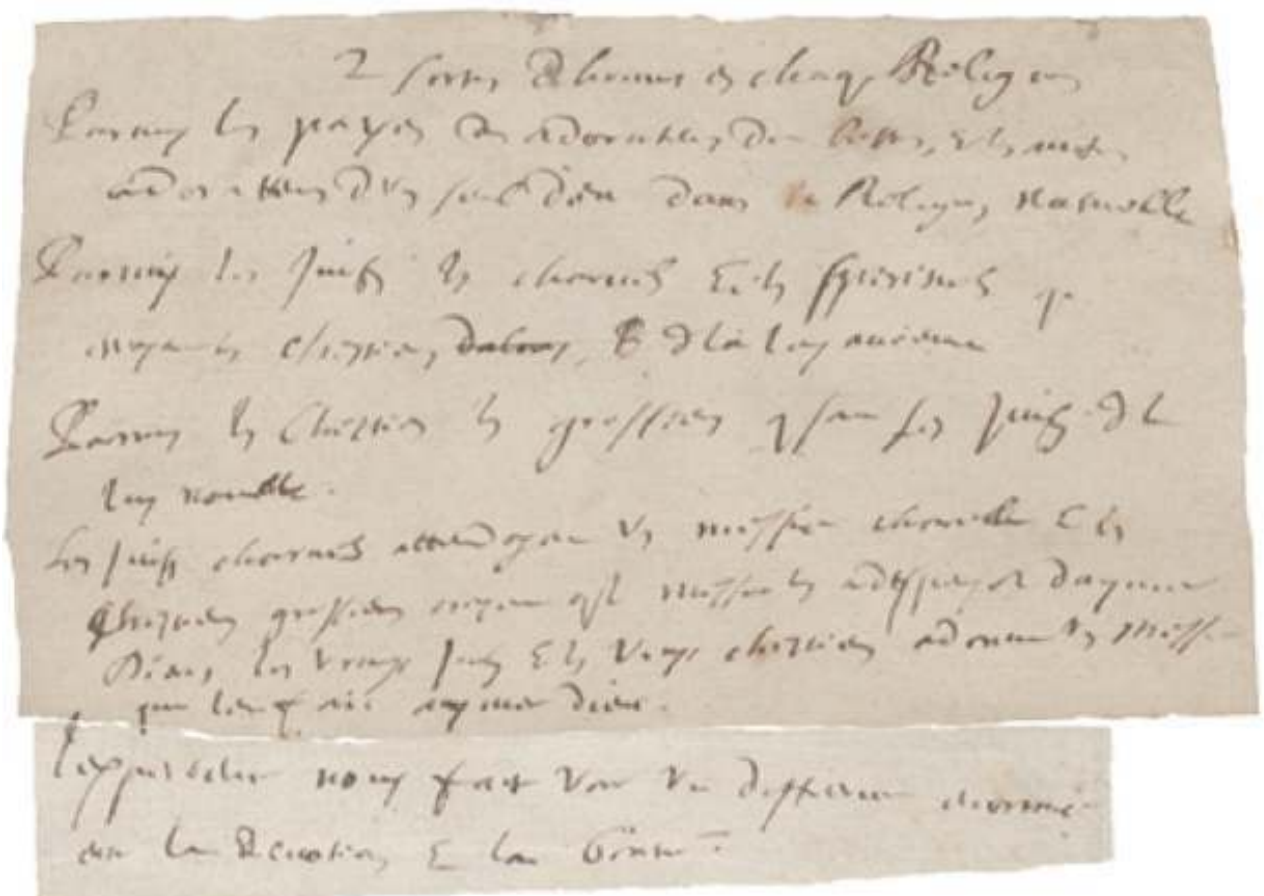

Cliché Pol Ernst 63.

64 Ce raboutage prouve, là encore, le rôle fondateur de l'exemple jésuite: dépassant l'exemple singulier de la Compagnie, qui tente de faire passer sa dévotion pour de la bonté, Pascal englobe dans sa dénonciation tous ceux que leur zèle papiste porte à condamner, sans même se pencher sérieusement sur le problème, les jansénistes qui refusent de se soumettre puis, en une généralisation absolue, tous ceux qui confondent zèle aveugle et bonté. Ainsi est confirmée l'origine polémique d'un thème apparemment pleinement apologétique, celui des «deux sortes d'hommes dans chaque religion »: la théorie des « charnels » ou " grossiers » opposés aux « spirituels », appliquée d'abord aux casuistes relâchés, s'étend désormais aux Juifs, signe de cette inflexion capitale. Le thème de l'amour de Dieu comme essence même du christianisme, fondamental dans la lutte des Provinciales, est récupéré à des fins apologétiques ${ }^{83}$.

À partir d'une critique du laxisme des casuistes, "chrétiens grossiers ${ }^{84}$ " trop dociles et soumis superstitieusement, qui font bon marché de la Chute pour en arriver à dispenser l'homme d'aimer Dieu, Pascal élabore donc dès cette seconde strate rédactionnelle un argument qui sera décisif dans son Apologie: le christianisme est la seule religion véritable, car, tenant compte de notre impuissance radicale, il prône l'amour exclusif de Dieu qui seul peut nous sauver:

Il faut donc que la vraie religion nous enseigne à n'adorer que lui et à n'aimer que

lui ${ }^{85}$.

Il faut n'aimer que Dieu et ne haïr que soi ${ }^{86}$.

Non seulement la dichotomie augustinienne entre amor Dei et amor sui rend parfaitement compte du cas des jésuites, qui recherchent la puissance temporelle et lui soumettent leur conduite spirituelle, mais il est fort possible que son usage apologétique chez Pascal ait été élaborée sur ce modèle même. S'appliquant également au peuple élu de l'Ancien 
Testament et, au-delà, à toute l'histoire de l'Église, ce schéma aboutit à intégrer la controverse apparemment spécialisée des Provinciales dans le cours de l'histoire universelle : « il en détermine la nécessité et la signification ${ }^{87}$ ».

De la même manière, le fragment 692-571, contemporain et ostensiblement référé à la querelle des Provinciales:

Montalte

Les opinions relâchées plaisent tant aux hommes qu'il est étrange que les leurs déplaisent. C'est qu'ils ont excédé toute borne. Et de plus il y a bien des gens qui voient le vrai et qui n'y peuvent atteindre, mais il y en a peu qui ne sachent que la pureté de la religion est contraire à nos corruptions. Ridicule de dire qu'une récompense éternelle est offerte à des mœurs escobartines.

trouve son prolongement et son accomplissement dans le fragment 633-526, également issu de la deuxième strate rédactionnelle, qui concerne le thème général de la condition humaine comme mi-lieu (apparu dès la première strate) :

Malgré la vue de toutes nos misères, qui nous touchent, qui nous tiennent à la gorge, nous avons un instinct que nous ne pouvons réprimer qui nous élève.

Si les jésuites sont voués à l'échec, c'est justement faute de ne pas prendre en compte cette ambivalence de l'homme, qui veut mais ne peut pas. Bien entendu, cette conception duelle de la condition humaine est loin d'être propre à Pascal ; cependant, l'importance qu'elle prendra dans son projet apologétique pourrait bien avoir été amorcée par l'utilisation polémique qu'il en a faite à la fin du combat des Provinciales. En ce sens, loin de ne constituer que des « répétitions » (au sens propre ou au sens théâtral du terme) des " petites lettres», ces fragments, prenant place au sein d'une réflexion plus ample, en sont le prolongement, réflexion critique d'une pensée sur elle-même grâce à l'utilisation d'un argument a simili.

69 À la fin de cette deuxième strate rédactionnelle, le projet apologétique est donc bien avancé. Comme le souligne P. Ernst, il serait certes exagéré d'y voir "l'esquisse d'un schéma d'ensemble ${ }^{88} "$, mais il est indéniable que des thèmes apologétiques capitaux commencent à se développer, touchant notamment à la conception du christianisme comme milieu. Plus généralement, dans l'Apologie, la critique des jésuites n'est bien sûr plus une fin en soi, mais un point de départ, souvent encore perceptible à ce stade : l'exemple de la Compagnie devient notamment le paradigme de la catégorie universelles des hommes "charnels ». La conception du rôle de la raison dans le domaine de la croyance subit enfin une inflexion majeure. Certes, de même qu'un bon usage de la raison était nécessaire pour établir une exégèse qui rende justice à la vérité des Écritures (c'est l'interprétation des préceptes évangéliques dans les Provinciales, l'interprétation des preuves prophétiques ou la méthode allégorique dans les Pensées), de même, quoique sur un plan bien différent, " la soumission et l'usage de la raison » doivent permettre d'éviter la superstition, c'est-à-dire de rendre justice à la vérité du christianisme. Cependant, le rôle de la raison dans ce second domaine est essentiellement réévalué à la baisse, de manière encore plus sensible que dans la strate précédente: l'accent est incontestablement placé sur la "soumission » plus que sur "l'usage » de la raison. Les prémisses, qui seront notamment rassemblées dans les liasses inaugurales "Commencement » et "Soumission et usage de la raison » sont donc posées, même si la forme de l'apologie reste encore indistincte ${ }^{89}$, puisque on peut penser que Pascal envisage encore éventuellement une présentation de son Apologie sous forme épistolaire, analogue à celle de ses polémiques « petites lettres ». 


\section{Un exemple d'évolution : le thème de l'honneur dans les Provinciales et dans les Pensées}

70 Une approche plus précise de l'élaboration progressive des grandes thématiques des Pensées ne peut s'entreprendre que point par point. Il n'est évidemment pas question d'envisager ici une étude exhaustive ; nous nous proposons plus modestement de prendre en compte un thème particulier, l'honneur, et d'en étudier la fortune au cours de l'élaboration progressive de l'Apologie. L'honneur est en effet un thème important dès les Provinciales - il est même au centre de la septième lettre; il réapparait également dès la première strate rédactionnelle de la future apologie $e^{90}$, puis dans chacune des strates suivantes, non sans subir tout au long de ce parcours des infléchissements significatifs. Cette place privilégiée n'est pas due au hasard: l'honneur est en effet un sujet central dans tous les manuels de casuistique du temps. Comme le remarque C. Chauchadis dans une étude consacrée au XVII ${ }^{e}$ siècle espagnol, mais qui s'applique également à la France (les jésuites français se réfèrent du reste souvent à leurs confrères casuistes d'outrePyrénées), «Il est [...] surprenant de comparer le peu de place qu'occupe la problématique de l'honneur dans l'Écriture Sainte et l'importance que lui donnent les moralistes du Siècle d'Or ${ }^{91}$ ».

Dans les Provinciales, l'honneur est essentiellement assimilé au point d'honneur, grandeur d'établissement qui ne repose que sur l'imagination. Certains fragments des Pensées, encore directement liés aux "petites lettres", reprennent cette association : l'honneur n'est envisagé qu'indirectement, sous l'angle du duel et de la casuistique : c'est le cas du fragment 722-602, appartenant à la strate dite "au cor ", tardive, mais qui comprend aussi des fragments recopiés, dont la rédaction initiale peut donc lui être bien antérieure - c'est vraisemblablement le cas ici. Ces fragments purement polémiques seront écartés en juin 1658.

72 Le problème de l'honneur est évoqué à plusieurs reprises, directement ou non, dans la liasse "Divertissement». Si l'honnête Montalte des Provinciales s'indignait de voir l'honneur aristocratique assimilé à un "bien" ${ }^{92}$ " qu'il faut défendre au besoin par la violence et le crime ${ }^{93}$, les Pensées confèrent à cette similitude de l'honneur et du bien une portée anthropologique plus large, en les présentant tous deux comme des formes particulières du divertissement commun à tous les hommes déchus :

Divertissement

On charge les hommes, dès l'enfance, du soin de leur honneur, de leur bien, de leurs amis, et encore du bien et de l'honneur de leurs amis. [...] Et on leur fait entendre qu'ils ne sauraient être heureux sans que leur santé, leur honneur, leur fortune et celles de leurs amis soient en bon état, et qu'une seule chose qui manque les rendra malheureux. [...] Voilà, direz-vous, une étrange manière de les rendre heureux. Que pourrait-on faire de plus pour les rendre malheureux ? Comment, ce qu'on pourrait faire? Il ne faudrait que leur ôter tous ces soins, car alors, ils se verraient, ils penseraient à ce qu'ils sont, d'où ils viennent, où ils vont ${ }^{94}$.

En outre, non seulement poursuivre son honneur comme un lièvre à la chasse est en soi un divertissement, mais les charges honorifiques, même une fois acquises, continuent à pourvoir indirectement l'homme en occasions de se divertir :

Divertissement [...]

Prenez-y garde, qu'est-ce autre chose d'être surintendant, chancelier, premier président, sinon d'être en une condition où l'on a le matin un grand nombre de 
gens qui viennent de tous côtés chez [eux] pour ne leur laisser pas une heure en la journée où ils puissent penser à eux-mêmes? Et quand ils sont dans la disgrâce et qu'on les renvoie à leurs maisons des champs, où ils ne manquent ni de biens ni de domestiques, [...] ils ne laissent pas d'être misérables et abandonnés parce que personne ne les empêche de songer à eux $^{95} \ldots$

Néanmoins, même la royauté, charge honorifique suprême, ne peut procurer le divertissement per se: le roi même est «plus heureux en s'attachant à ses vains amusements qu'à la vue de sa grandeur ${ }^{96}$ ». L'honneur humain est donc certes une illusion, mais il s'agit d'un leurre nécessaire, non seulement aux nobles, mais à tout homme dans l'état post-lapsaire :

ceux qui font sur cela les philosophes et qui croient que le monde est bien peu raisonnable de passer tout le jour après un lièvre qu'ils ne voudraient pas avoir acheté ne connaissent pas notre nature ${ }^{97}$.

Si sa poursuite est un mal, ce n'est pas seulement parce qu'elle peut conduire à la violence et au meurtre, c'est parce qu'elle empêche l'homme de contempler sa misère pour se tourner enfin vers Dieu.

Pour autant, les fragments les plus nombreux et les plus explicites concernant l'honneur ne sont pas rangés dans la liasse "Divertissement ${ }^{98}$ "; ils appartiennent à une strate tardive $^{99}$, qui porte en particulier les fragments $89-123,90-124$ et 92-126; tous intitulés « Raison des effets » et rangés ensuite dans la liasse correspondante, ils témoignent d'une étape plus avancée dans la rédaction de l'Apologie, au sein de laquelle l'honneur est inséré dans la dynamique herméneutique progressant de l'opinion du peuple à celle des dévots, en passant par celles des demi-habiles et des habiles. Dans l'ordre de rédaction reconstitué par P. Ernst ${ }^{100}$ (cliché A 169), le fragment 92-126 vient d'abord, avec une première méditation - encore un peu embryonnaire - sur «les opinions du peuple saines » :

Il est donc vrai de dire que tout le monde est dans l'illusion, car encore que les opinions du peuple soient saines, elles ne le sont pas dans sa tête. [...] Il est vrai qu'il faut honorer les gentilshommes, mais non pas parce que la naissance est un avantage effectif, etc.

Le fragment $90-124$, rédigé ensuite ${ }^{101}$, poursuit la réflexion en introduisant, à propos du même exemple (dont le caractère emblématique est souligné par la répétition des antithèses entre mépriser et honorer), la gradation entre l'opinion du peuple et celle des «chrétiens parfaits» :

Le peuple honore les personnes de grande naissance. Les demi-habiles les méprisent, disant que la naissance n'est pas un avantage de la personne, mais du hasard. Les habiles les honorent, non par la pensée du peuple, mais par la pensée de derrière. Les dévots, qui ont plus de zèle que de science, les méprisent, malgré cette considération qui les fait honorer par les habiles, parce qu'ils en jugent par une nouvelle lumière que la piété leur donne. Mais les chrétiens parfaits les honorent par une lumière supérieure.

Enfin, après le long fragment 93-127, le feuillet s'achève sur la référence aux Essais, non évoqués jusque-là sur ce point :

Cela est admirable : on ne veut pas que j'honore un homme vêtu de brocatelle, et suivi de sept ou huit laquais. Et quoi, il me fera donner les étrivières, si je ne le salue. [...] Montaigne est plaisant de ne pas voir quelle différence il y a, et d'admirer qu'on y en trouve, et d'en demander la raison ${ }^{102}$. 


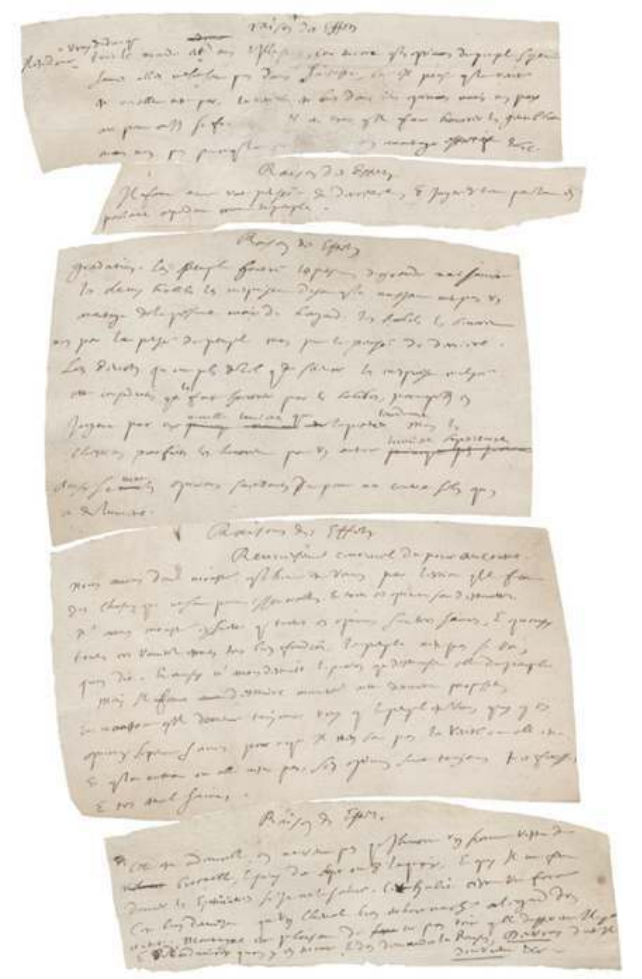

Cliché Pol Ernst 169. vaine qu'idolâtre; il est intégré au sein d'une chaîne de causalité socio-politique plus complexe, où il trouve une forme de justification - fût-elle partielle :

Cet habit, c'est une force ${ }^{103}$. ulement par construction a posteriori, « parce qu'il est injuste de [le] troubler ${ }^{107}$ »-c'est l'interprétation de L. Thirouin ${ }^{108}$, reste que le dernier mot, l'ultime sagesse, revient en définitive à ceux qui savent rendre aux grandeurs humaines les honneurs qui leur reviennent, en considération de l'ordre que Dieu a établi sur terre après la Chute, ordre qui est à la fois punition par son absurdité même, et moyen de rachat par sa justification 
supérieure ${ }^{109}$. L'honneur, pris dans une acception plus large que dans les Provinciales, qui l'associaient surtout à la casuistique du duel, n'est donc plus seulement dénoncé comme désordre, mais au contraire réinséré dans un ordre moins apparent, mais plus essentiel.

Mieux : le fondement même de l'honneur, à savoir l'amour-propre, en soi principe de désordre, verra sa force détournée au profit du bien commun, et Pascal pourra admirer «la grandeur de l'homme, d'avoir tiré de la concupiscence un si bel ordre ${ }^{110}$ ». Si l'honneur est le plus précieux des biens, ce n'est pas seulement parce que des avantages effectifs y sont attachés; c'est aussi parce qu'il est essentiel à un fonctionnement social étroitement dépendant des signes, ces signes mêmes que revendique et défend le sens de l'honneur : contre le "moraliste demi-habile», Pascal soutient que "cette superficialité indéniable de la vie sociale n'est pas déraisonnable, mais [...] signifie avec précision et efficacité les mécanismes essentiels qui garantissent la communauté111 ». Si ces signes sont remis en question impunément, c'est l'anarchie qui règne, la « confusion » redoutée au fragment 80 : «La mutuelle dépendance des hommes impose qu'ils reconnaissent et saluent à l'envi les uns dans les autres leur royauté de concupiscence ${ }^{112}$. »

Ainsi, à partir de ce qui pouvait apparaitre comme une dispute théologique byzantine réservée à des spécialistes, Pascal élabore progressivement un projet apologétique aussi cohérent que novateur, s'étendant petit à petit à tous les domaines humains. L'étude des fragments rédigés à l'époque des Provinciales permet de préciser la manière dont il nait et se précise au cours d'une période cruciale allant de l'automne 1656 au printemps 1657 . Si des thèmes proprement théologiques tels que le Dieu caché ou la théorie des trois états ne trouvent peut-être qu'une illustration particulièrement pertinente dans la dispute avec les jésuites, sans être à proprement parler suscités par elle, des problématiques plus anthropologiques, comme la réflexion pascalienne sur la justice ou la tyrannie pourraient bien être nées de la polémique même des " petites lettres ». S'agissant d'un thème comme celui des Juifs charnels, il est en revanche malaisé de déterminer s'il a pris naissance dans le contexte de la polémique, ou s'il lui préexistait et lui a été adapté.

Dans cette perspective, les Provinciales ne vaudraient-elles que comme annonciatrices de la future Apologie? Ce serait oublier qu'offrant un point de vue original sur la pensée pascalienne, grâce à leur appartenance générique et au rapport particulier qu'elles entretiennent avec le lecteur, elles permettent de contrebalancer (et non de contredire) de façon décisive ce qu'on a pu appeler le "pessimisme» des Pensées (ainsi le rôle problématique dévolu à la raison, à la justice humaine), et d'approcher ainsi au plus près une pensée complexe et nuancée, dès ses prémisses mêmes.

La comparaison globale des deux œuvres, que nous n'avons bien sûr pas prétendu entreprendre ici, permettrait sans doute de mettre en lumière la manière dont la pensée pascalienne se déploie à partir de ces thématiques fondamentale, se nuance, se généralise, ou au contraire se modalise, selon le public auquel Pascal s'adresse et le but qu'il poursuit. Les mêmes thèmes s'y retrouvent, comme en des variations musicales, mais infléchis différemment, si différemment parfois qu'on a pu soupçonner, de manière plus ou moins explicite, une rupture entre les deux œuvres, postulat toujours coûteux dans une démarche critique.

Pourtant, au-delà de la différence des publics et des objectifs, qui peut expliquer cette méprise, ces deux textes partagent un même élan, celui d'appels à la conversion, dans tous les sens du terme, qui était alors au cœur des préoccupations de Pascal. Comme le souligne en effet J. Laporte, «l'apologétique ne sert pas seulement aux incrédules. [...] Elle 
sert aussi [...] aux chrétiens, qu'elle met en mesure [...] de rendre compte de leur foi à euxmêmes et aux autres ${ }^{113} »$. Pour H.Gouhier ${ }^{114}$, cette interprétation est confirmée par l'opuscule publié sous le titre de Comparaison des chrétiens des premiers temps avec ceux d'aujourd'hui ${ }^{115}$, antérieur aux "petites lettres» selon Havet ${ }^{116}$, plus ou moins contemporain des Provinciales et des premières Pensées si l'on se réfère à l'hypothèse de L. Lafuma (« entre 1655 et $\left.1657^{117} »\right)$, un peu plus tardif selon celle, plus vraisemblable, de $\mathrm{J}$. Mesnard, qui le situe probablement en 1657, ou à la rigueur dans la première moitié de $1658^{118}$, en tout cas à peu près en même temps - et cela est significatif - que l'Écrit sur la conversion du pêcheur ${ }^{119}$. Dans la lignée de ses amis de Port-Royal, plus saint-cyraniens que jansénistes à proprement parler, Pascal développe ainsi une spiritualité et une écriture de la conversion, du refus de l'amour de soi au profit de l'amour de Dieu. Ce qu'il attaque avant tout dans la morale des jésuites, c'est, beaucoup plus que certaines décisions relâchées des casuistes, l'esprit dont ceux-ci procèdent, l'attitude spirituelle qu'ils tendent à susciter, voire encouragent, chez les chrétiens : en admettant qu'il est permis de choisir en fonction de son intérêt entre plusieurs opinions probables, ils se comportent comme si faire le bien ne nécessitait pas une conversion du cœur, un passage radical de l'amour-propre à l'amour de Dieu. Le chemin à parcourir par l'incroyant, et auquel l'invitent les Pensées, est certes plus long, mais il n'est pas fondamentalement différent: dans la perspective augustinienne en effet, la négation de Dieu n'est qu'une forme outrée de l'affirmation de soi, tout comme l'affirmation de Dieu est une forme de négation de soi. La conversion de l'incroyant n'est donc qu'une variation de celle du chrétien, et doit d'ailleurs, pour être complète, se confondre finalement avec elle : l'apologétique, comme la morale, se prolonge nécessairement en spiritualité. Ainsi, loin de trahir Montalte, Salomon de Tultie prolonge sa réflexion, l'amplifie et l'approfondit, tout en en infléchissant la présentation, de manière à toucher un public plus large, qui rassemble croyants peu fervents et incroyants dans une même tension vers la conversion.

\section{NOTES}

1. Plus généralement, seuls quarante-cinq dossiers sur les soixante et un que comptent les Pensées appartiennent pleinement à la future Apologie, Pascal ayant mis de côté (peut-être provisoirement dans certains cas) trois liasses sur les miracles, une liasse intitulée "Contre la fable d'Esdras » et douze dossiers de « Pensées mêlées ».

2. B. Pascal, Les Provinciales, éd. L. Cognet et G. Ferreyrolles, Paris, Bordas, 1992, III ${ }^{\mathrm{e}}$ lettre, p. 51.

3. B. Pascal, Pensées, éd. Ph. Sellier, Paris, Garnier, 1999, fr. 56-89. Dans les références des fragments des Pensées, le premier numéro indiqué renvoie à l'édition Lafuma et le second à l'édition Sellier.

4. Pensées, fr. 904-450.

5. Pensées, fr. 909-451.

6. Ils sont numérotés XXXII, XXXIII et XXXIV dans l'édition Lafuma; XXX, XXXI et XXXII dans l'édition Sellier.

7. De même, les exemples de notes liées aux écrits connexes des Provinciales (Projet de Mandement, Écrits des Curés...), donc souvent légèrement postérieures à celles-ci, ne manquent pas. Cf. G. 
Bottereau, « Notes de lecture de Blaise Pascal sur les jésuites », Archivium Historicum Societatis Jesu, $\mathrm{n}^{\circ}$ XLIII, 1974, pp. 147-156.

8. P. Nicole, Lettre au marquis de Sévigné (date inconnue), cité par L. Lafuma dans Pascal. CEuvres complètes, Paris, Seuil, 1963, t. III : «Documents", p. 198. Sur la difficulté de déterminer les fragments destinés à la future Apologie, cf. Roger E. Lacombe, L'Apologétique de Pascal. Étude critique, Paris, Presses Universitaires de France, 1958, p. 12 sq.

9. Cf. L. Goldmann, Le Dieu caché. Étude sur la vision tragique dans les Pensées de Pascal et dans le théâtre de Racine, Paris, Gallimard, 1955, p. 165 ; G. Ferreyrolles, « Un âge critique : les trente ans du Dieu caché », Commentaire, n 34, 1986, pp. 290-297 et « Goldmann visionnaire », « Port-Royal au miroir du XX ${ }^{\text {ème }}$ siècle ", Chroniques de Port-Royal, n 49, 2000, pp. 71-96.

10. H. Gouhier n'hésite pas à faire remonter l'intention apologétique de Pascal à la "première conversion » de 1646, et Ph. Sellier en décèle une trace dans les ardentes discussions de Pascal avec Antoine de Rebours, en 1648 (Pascal, Pensées, éd. citée, introduction, p. 14) : il y aurait donc continuité entre les diverses œuvres religieuses de Pascal. Quant à l'impulsion apologétique fondamentale, elle est commune aux Provinciales et aux Pensées, puisqu'il s'agit incontestablement de la « nuit de feu » et de la « seconde conversion » de 1654.

11. Cf. H. Gouhier, Blaise Pascal. Conversion et apologétique, Paris, Vrin, 1986.

12. P. Ernst, Géographie et stratigraphie des Pensées de Pascal, Oxford, Voltaire Foundation, 1996.

13. Mieux encore, la majorité de ceux-ci ont été écrits sur quatre types seulement de papier, qui correspondent pour P. Ernst aux quatre «strates majeures ».

14. Cf. P. Ernst, op. cit., p. 242.

15. Cf. Ph. Sellier, "De la tyrannie ", in Justice et force. Politique au temps de Pascal, Colloque de Clermont-Ferrand (1990), Paris, Klincksieck, 1995, pp. 365-375. Dans cet article, modèle critique et méthodologique pour la présente étude, $\mathrm{Ph}$. Sellier a le premier approfondi un concept pascalien en intégrant les données chronologiques mises au jour par P. Ernst.

16. Cf. en particulier la citation tot et tanta mala pacem (Sagesse, 14, 22), présente dans le fragment et commentée, dans un contexte très similaire, dans le Second écrit des curés de Paris, dont le fragment est très probablement, comme le remarque Ph. Sellier, un "avant-texte " (Pascal, Pensées, éd. citée, p. 410, n. 44).

17. Cf. par exemple le passage du Factum : "Voilà le comble de l'insolence où les Jésuites ont élevé les casuistes. Après avoir abusé de la modération des ministres de l'Église pour introduire leurs opinions impies, ils sont aujourd'hui arrivés à vouloir chasser du ministère de l'Église ceux qui refusent d'y consentir» (Appendice de l'éd. citée des Provinciales, p. 415). Elle est présente dans le fragment 700-578 sous la forme : «Non seulement ils veulent être soufferts dans l'Église, mais comme s'ils étaient devenus les plus forts, ils en veulent chasser ceux qui n'en sont pas. »

18. Selon J. Mesnard, c'est à la fin du mois d'avril ou au début du mois de mai 1657 que Pascal entreprend la rédaction d'une lettre au P. Annat, qui aurait pu constituer une dix-neuvième lettre. Cf. Pascal, CEuvres complètes, éd. J. Mesnard, t. III, « CEuvres diverses (1654-1657), Paris, Desclée de Brouwer, 1991, p. 466.

19. Sur cette question, et sur le statut du miracle dans l'apologétique pascalienne, cf. T. Shiokawa, Pascal et les miracles, Paris, Nizet, 1977, chap. IV et V.

20. G. Périer, La Vie de M. Pascal, in Pascal. Cuvres complètes, op. cit., t. I, pp. 583-584. En fait, Gilberte passe sous silence l'exploitation polémique du miracle pour n'en retenir que son rôle de déclencheur dans le projet apologétique, rôle qui, comme le montre T. Shiokawa est essentiellement indirect, l'argument du miracle lui-même étant très tôt écarté de l'Apologie (ib., pp. 179 sq.).

21. H. Gouhier, Commentaires, Paris, Vrin, 1966, p. 183.

22. Cf. IV lettre aux Roannez, in Pascal, Euvres complètes, éd. citée, t. III, 1991, p. 1035-1036. Rappelons que pour Ph. Sellier, c'est la liturgie du Saint-Sacrement qui imprègne cette lettre et a $\mathrm{pu}$ jouer un rôle matriciel dans la naissance de ce thème: la comparaison entre le voile de 
l'humanité et celui de l'Eucharistie est présente dans l'hymne et les litanies qu'on y chante ou psalmodie, la neuvième litanie portant le titre significatif de «Deus absconditus et salvator ». Cf. Ph. Sellier, Pascal et la liturgie, Paris, Presses Universitaires de France, 1966, p. 69. Cf. aussi sur ce point H. Michon, « Deus absconditus ", XVII siècle, nº 177, 1992-4.

23. Pensées, fr. 225-258.

24. Cf. G. Ferreyrolles, Pascal et la raison du politique, Paris, Presses Universitaires de France, 1984, chap. IV. «La loi naturelle » et, du même auteur, "La loi naturelle dans les Provinciales et les Pensées", in "Le droit à ses époques». De Pascal à Domat, CD-Rom, Presses Universitaires Blaise Pascal, 2003.

25. Cf. T. Shiokawa, op.cit., p. 158, n. 129.

26. Pour une étude plus détaillée des interrogations posées par cette figure particulièrement problématique, je me permettrai de renvoyer à mon article intitulé «La figure du juge dans les Provinciales et dans les Pensées : rupture ou continuité ?", in Pascal / New Trends in Port-Royal Studies, Actes du $33^{e}$ congrès annuel de la North American Society for Seventeenth-Century French Literature (may 2001), t. I, Tübingen, Gunter Nach Verlag, 2002.

27. T. Ishikawa, «La théorie des trois états de l'homme chez Pascal », Équinoxe (Kyoto), n 6, été 1990, pp. 117-142.

28. Cf. G. Ferreyrolles, ib. et Ph. Sellier, Pascal et saint Augustin, Paris, A. Colin, 1970, chap. I.7 « Loi naturelle et obscurcissement chez Pascal ».

29. L'expression est de $\mathrm{T}$. Ishikawa, $i b$.

30. Elle apparaît certes sous une forme embryonnaire dans les $\mathrm{IV}^{\mathrm{e}}$ et $\mathrm{VI}^{\mathrm{e}}$ lettres à $\mathrm{M}^{\mathrm{lle}}$ de Roannez, mais les "gens du monde", plus adaptés à la destinataire, remplacent les Juifs; la réflexion pascalienne sur l'économie proprement historique du salut ne semble pas réellement engagée, au profit d'une méditation sur un salut purement individuel. Surtout, la nature propre des chrétiens n'est pas problématisée comme telle : les chrétiens se subdivisent entre hérétiques et catholiques sans que leur état lui-même, milieu entre les Juifs et les bienheureux, ne soit véritablement défini. Pascal possédait-il déjà intégralement sa théorie tripartite et jugeait-il inopportun de l'exposer intégralement à son amie ? Ou, plus probablement compte tenu de son intense activité intellectuelle à cette période de menaces et de tensions, ses réflexions ont-elles mûri tout au long du second semestre 1656 , pour parvenir à leur formulation définitive entre la VI lettre à $\mathrm{M}^{\mathrm{lle}} \mathrm{de}$ Roannez et la XVI ${ }^{\mathrm{e}}$ Provinciale, soit pendant le mois de novembre 1656 ?

31. Ce lien réapparaîtra, par exemple, dans le fragment 826-667, dont la datation demeure problématique.

32. Provinciales, éd. citée, $\mathrm{XVI}^{\mathrm{e}}$ lettre, p. 314.

33. Même si, en un sens, Dieu « s'est encore plus caché en se couvrant de l'humanité » (IV lettre à $\mathrm{M}^{\text {lle }}$ De Roannez, in Pascal, CEuvres complètes, éd. citée, t. III, p. 1035).

34. Pensées, fr. 517-452.

35. Pensées, fr. 519-453.

36. Pensées, fr. 516-452.

37. Ce thème réapparaîtra dans la Prière pour demander à Dieu le bon usage des maladies, mais la souffrance remplacera les figures, et la consolation la vérité.

38. Cf. H. Michon, « Aristote et Pascal », Courrier du Centre International Blaise Pascal, 2002, $\mathrm{n}^{\circ} 24$, p. 24.

39. Provinciales, éd. Citée, IV lettre, p. 60.

40. Pensées, fr. 101-134, strate non identifiée.

41. D. Descotes, «La calomnie dans Les Provinciales », Courrier du Centre International Blaise Pascal, 1996, n 18, p. 14.

42. Pensées, fr. 962-696.

43. L'opposition aux erreurs symétriques des pyrrhoniens et des stoïciens n'est bien sûr pas nouvelle: elle date de l'Entretien, dont P. Courcelle a montré qu'il avait pu servir à Pascal de 
document de travail lorsqu'il entreprit son Apologie (L'Entretien de Pascal et Sacy. Ses sources et ses énigmes, Paris, Vrin, 1960, p. 149). Mais sa formalisation définitive, et son lien avec le thème du milieu, ne seront élaborés que progressivement.

44. D. Descotes, ibid., p. 21.

45. Provinciales, éd. citée, XVIII ${ }^{\mathrm{e}}$ lettre, p. 374. Cf. aussi la XVII lettre, ib., p. 343 : «Dieu conduit l'Église, dans la détermination des points de la foi, par l'assistance de son esprit, qui ne peut errer ; au lieu que, dans les choses de fait, il la laisse agir par les sens et par la raison, qui en sont naturellement les juges. » Sur la conception pascalienne du rôle des sens, cf. T. Shiokawa, Pascal et les miracles, Paris, Nizet, 1977, p. 53 sq.

46. Il figurait à l'origine en marge du fragment 278-309, intitulé «Péché originel », qui sera ensuite classé dans la liasse intitulée « Rabbinage ».

47. On peut cependant rappeler que selon $\mathrm{H}$. Gouhier, l'Entretien avec M. de Sacy déjà, loin de défendre une certaine validité de la philosophie, use au contraire du scepticisme montaignien pour « discréditer toute apologétique qui inviterait la foi à faire un bout de chemin avec la raison ou plus exactement à se taire provisoirement pour laisser la raison cheminer, seule, jusqu'au moment de l'inévitable relève » (Blaise Pascal. Commentaires, Paris, Vrin, 1984, p. 90). Toutefois, si H. Gouhier voit dans cet entretien une préfiguration capitale du projet apologétique, J. Mesnard et $\mathrm{P}$. Mengotti-Thouvenin nuancent de manière décisive cette interprétation : ce qui séparerait l' Entretien des Pensées, ce serait justement la querelle dramatique des Provinciales, qui assombrit la joie du jeune converti de 1654 (Pascal, Entretien avec M. De Sacy, Paris, Desclée de Brouwer, 1994, Présentation, p. 75).

48. Pensées, fr. 516-452. Il n'est peut-être pas indifférent que le refus des règles aussi rassurantes que fausses soit d'emblée associé, dans le même fragment, au refus d'un style conventionnel ; le fragment cité plus haut commençait en effet par une remarque proprement stylistique : «Quand dans un discours se trouvent des mots répétés et qu'essayant de les corriger on les trouve si propres qu'on gâterait le discours, il les faut laisser, c'est la marque. Et c'est là la part de l'envie, qui est aveugle et qui ne sait pas que cette répétition n'est pas faute en cet endroit. Car il n'y a pas de règle générale" (Pensées, fr. 515-452). Décrets abusifs du pape, juridiction rigide des législateurs des belles-lettres, sont en quelque sorte disqualifiés dans un même élan.

49. Cf. L. Thirouin, "L'Éthos de Montalte dans les Provinciales », in Ethos et pathos. Le statut du sujet rhétorique. Actes du Colloque international de Saint-Denis (19-21 juin 1997)), éd. R. Lockwood, Paris, Champion, 2000.

50. Cf. Ph. Sellier, "Vers l'invention d'une rhétorique: les Provinciales", in R. Duchêne, L'Imposture littéraire dans les Provinciales de Pascal, Marseille, Université de Provence, 1985, pp. 348.

51. P. Ernst, op. cit., p. 209.

52. Pensées, fr. 232-264. Cf. aussi fr. 228-260, 232-264, 233-265, 234-266, 235-267, 237-269. Le célèbre fragment A.P.R. (149-182), manifestement apologétique, développe la même idée ; or, on peut le considérer comme contemporain des dernières Provinciales, puisqu'il se rapporte vraisemblablement à la conférence qu'Étienne Périer qualifie dans la Préface de 1669 d'« occasion qui se rencontra il y a environ dix ou douze ans » (Les «Pensées» de Port-Royal, Préface, in B. Pascal, Euvres complètes, éd. M. Le Guern, Paris, Gallimard, 2000, t. II, p. 902).

53. P. Force, Le problème herméneutique chez Pascal, Paris, Vrin, 1989, p. 151 sq.

54. Les Provinciales, éd. citée, VI lettre, pp. 100-101.

55. L'utilisation abusive du sens figuré est une critique couramment portée contre les protestants. Cf. Bossuet, L'Histoire des variations...

56. Pensées, fr. 257-289

57. Cf. P. Force, op.cit., p. 159 sq.

58. Rappelons que pour Ch. Perelman et L. Olbrechts-Tyteca, «la pétition de principe, qui ne concerne pas la vérité, mais l'adhésion des interlocuteurs aux prémisses que l'on présuppose, 
n'est pas une faute de logique, mais de rhétorique; elle se comprend, non à l'intérieur d'une théorie de la démonstration, mais par rapport à la technique argumentative. En fait, la pétition de principe consiste en un usage de l'argument ad hominem lorsqu'il n'est pas utilisable, parce qu'elle suppose que l'interlocuteur a déjà adhéré à une thèse que l'on s'efforce justement de lui faire admettre » (Traité de l'Argumentation, La nouvelle rhétorique, Bruxelles, Éditions de l'université de Bruxelles, 1976 [1970], § 28, l'argumentation « ad hominem », p. 151).

59. Pensées, 260-291.

60. Provinciales, éd. citée, XVII ${ }^{\mathrm{e}}$ lettre, p. 338.

61. Provinciales, éd. citée, XVIII lettre, p. 376. Sur cette question, cf. P. Force, Le problème herméneutique chez Pascal, Paris, Vrin, 1989, pp. 159 sq.

62. Cf. Provinciales, IV lettre. Cf. aussi G. Ferreyrolles, Les reines du monde. L'imagination et la coutume chez Pascal, Paris, Champion, 1995, pp. 32 sq.

63. Pensées, fr. 588-487.

64. Pensées, fr. 284-316.

65. , Appendice de l'éd. citée des Provinciales, pp. 412 sq.

66. Cf. Ph. Sellier, art. cité.

67. Pour Pascal en effet, le pape a certes le droit de condamner cinq propositions hérétiques sur la grâce, mais nullement de soutenir qu'elles se trouvent dans l'Augustinus.

68. Cf. Provinciales, éd. citée, XVII lettre, p. 350.

69. Cf. notamment le fragment 704-582, rédigé entre la XVII ${ }^{\mathrm{e}}$ et la XVIII ${ }^{\mathrm{e}}$ Provinciales. Cf. G. Ferreyrolles, "Pascal et le modèle vénitien », in Justice et force. Politique au temps de Pascal, colloque de Clermont-Ferrand (1990), Paris, Klincksieck, 1995.

70. Provinciales, éd. citée, XVIII ${ }^{\mathrm{e}}$ lettre, p. 376.

71. Pensées, fr. 173-204. Cf. fragment 181-212 cité précédemment.

72. Pensées, fr. 179-210.

73. Provinciales, éd. citée, XVIII ${ }^{\mathrm{e}}$ lettre, p. 375.

74. Pensées, fr. 364-396.

75. Cf. H. Gouhier, Commentaires, p. 183, même si d'autres indications tendent à laisser prévoir un livre plus traditionnel, divisé en chapitres ou en parties, avec des préfaces, etc.

76. Pensées, fr. 576-479.

77. Pensées, fr. 219-252.

78. $\mathrm{La} \mathrm{X}^{\mathrm{e}}$ lettre, qui porte l'indignation : «on ne peut ouïr sans horreur les choses que je viens d'entendre " paraît également avoir inspiré la note 849-430, jetée sur une grande feuille au milieu de notes destinées à l'écrit sur les miracles qui aurait fait rebondir la polémique des Provinciales dans une nouvelle direction, et qu'on peut dater de la fin de l'automne 1656, au cours duquel Pascal a approfondi sa réflexion sur les miracles : «Dire que Jésus-Christ qui est venu ôter les figures pour mettre la vérité ne soit venu que mettre la figure de la charité pour ôter la réalité qui était auparavant, cela est horrible ».

79. Pascal, Second Écrit des Curés de Paris, appendice à l'éd. citée des Provinciales, p. 424.

80. Cf. J. Mesnard, op. cit., t. I, p. 190.

81. Appendice de l'éd. citée des Provinciales, p. 463.

82. Cf. D. Descotes, «Piège et paradoxe chez Pascal», in Méthodes chez Pascal, Paris, Presses Universitaires de France, 1979, p. 512.

83. Cette bipartition ne correspond nullement à un rejet de la théorie des trois états, comme en témoigne par exemple le fragment 75-110, appartenant lui aussi à cette seconde strate rédactionnelle.

84. Pensées, fr. 286-318. Cf. aussi le fragment 287-319, qui est plus tardif - il appartient à la strate rédactionnelle suivante ( $\mathrm{FNPH} »)$.

85. Pensées, fr. 205-237.

86. Pensées, fr. 373-405. 
87. D. Descotes, introduction à l'édition des Pensées, Paris, Garnier-Flammarion, 1976.

88. P. Ernst, op. cit., p. 242.

89. Selon Ph. Sellier, ces liasses réunissent des notes destinées à une ample ouverture de l'apologie, vraisemblablement, du moins dans le projet initial («L'ouverture de l'apologie pascalienne ", XVII siècle, $\left.\mathrm{n}^{\circ} 177,1992-4\right)$; or une part non négligeable des fragments qui constitueront ces futures liasses date précisément de l'époque des Provinciales.

90. P. Ernst, ibid.

91. C. Chauchadis, Morale, Honneur et Société dans l'Espagne de Philippe II, Paris, Éditions du C.N.R.S., 1984, p. 76.

92. Cf. Provinciales, éd. citée, VII lettre, p. 123. «On peut ainsi courir après son honneur, comme après du bien dérobé ».

93. En fait, la plupart des casuistes proscrivaient la vengeance directe et individuelle et prônaient, à défaut du pardon, l'appel à la justice temporelle. Escobar, qui accepte la vengeance à condition qu'elle soit dépourvue de haine, représente une position extrême.

94. Pensées, fr. 139-171.

95. Pensées, fr. 136-168.

96. Pensées, fr. 137-169.

97. Ibid.

98. La réflexion sur le divertissement sera essentiellement élaborée à partir d'une relecture des liasses «Misère ", "Ennui » et "Vanité » à la lumière de l'herméneutique développée dans la liasse « Raison des effets ». Voir L. Thirouin, « Raison des effets, essai d'explication d'un concept pascalien », XVII ${ }^{e}$ siècle, 1982, n 132, pp. 31-50.

99. Celle que $\mathrm{P}$. Ernst appelle « FNIC », et dont il situe la rédaction après avril 1658.

100. P. Ernst, op. cit., pp. 392-395.

101. Le fragment 91-125 vient s'intercaler entre les deux.

102. Pensées, fr. 89-123. Rappelons que pour Aristote, l'honneur est le plus grand des biens extérieurs.

103. Pensées, fr. 89-123.

104. Pensées, fr. 90-124.

105. Pascal. Cuuvres complètes, éd. J. Mesnard, éd. citée, t. IV, p. 1032.

106. G. Ferreyrolles, Pascal et la raison du politique, Paris, Presses Universitaires de France, 1984, p. 162.

107. Pascal, Trois discours..., ibid.

108. L. Thirouin, Le Hasard et les Règles, Paris, Vrin, 1991, pp. 57 sq.

109. Cf. Provinciales, XIV lettre, éd. citée, p. 268 ; Pensées, fr. 106-138 et 118-150.

110. Pensées, fr. 106. Pour L. Thirouin, cette admiration est cependant purement esthétique (« Pascal et le savoir-vivre (esthétique de la vie sociale) », in Pascal Port-Royal Orient Occident, Paris, Klincksieck, 1991, p. 272).

111. L. Thirouin, ibid., p. 270.

112. G. Ferreyrolles, ibid., p. 140. G. Ferreyrolles souligne à juste titre qu'il n'est pas jusqu'à l'économie - avec ses retentissements sur les conditions de vie des hommes, en l'occurrence surtout des femmes - qui ne bénéficie de cet attachement à l'honneur ; en imposant aux nobles un certain train de vie, donc des dépenses, il stimule l'artisanat du pays.

113. J. Laporte, Le cœur et la raison selon Pascal, Paris, Elzévir, 1950.

114. H. Gouhier, Blaise Pascal. Conversion et apologétique, Paris, Vrin, 1986, p. 129.

115. Il n'est pas indifférent que les copies par lesquelles seules on connaît aujourd'hui ce texte soient intitulées, l'une Réflexions sur la manière dont on était autrefois reçu dans l'Église, comme on y vivait, comme on y entre et on y vit aujourd'hui, et l'autre, de manière encore plus significative si l'on songe aux Provinciales, Quelles sont les causes de la nonchalance et du peu d'instruction des chrétiens d'aujourd'hui? 
116. Éd. des Pensées, 1852, p. 474, n. 1. Comme le souligne J. Mesnard, «tous les commentateurs qui ont repris le problème n'ont fait que suivre, expressément ou non, l'opinion de Havet » ( op. cit., t. IV, p. 52).

117. Pascal, Euvres complètes, éd. L. Lafuma, Paris, Seuil, p. 361.

118. J. Mesnard, ib., pp. 52-53.

119. Avec T. Shiokawa, J. Mesnard souligne que cet écrit traite avant tout de la signification du baptême, étroitement liée à la conversion : « De l'une et de l'autre il est question simultanément dans les textes de la session VI du concile de Trente auxquels songeait sans doute " Pascal (ibid, p. 53). Un peu plus tard, la Prière pour demander à Dieu le bon usage des maladies s'inscrira elle aussi dans cette direction fondamentale. J. Mesnard souligne que Pascal s'inspira pour l'écrire de la Prière pour demander à Dieu la grâce d'une véritable et parfaite conversion de G. Le Roy (ib., p. 979).

INDEX

Mots-clés : Pascal, Pensées, Provinciales, conversion, honneur Keywords : honour

\section{AUTEUR}

\section{ANNE RÉGENT}

Université Paris III - Sorbonne Nouvelle 